\title{
TITLE: HYDRODYNAMIC INSTABILITIES IN INERTIAL CONFINEMENT FUSION
}

AUTHOR(S): Nelson M. Hoffman

SUBMITTEd TO: 45 th Scottish Universities Summer School in Physics, University of St. Andrews,
St. Andrews, Scotland, UK, 7 - 20 August 1994 DISCLAIMER

This report was prepared as an account of work sponsored by an agency of the United States Government. Neither the United States Government nor any agency thereof, nor any of their employees, makes any warranty, express or implied, or assumes any legal liability or responsibility for the accuracy, completeness, or usefulness of any information, apparatus, product, or process disclosed, or represents that its use would not infringe privately owned rights. Reference herein to any specific commercial product, process, or service by trade name, trademark, manufacturer, or otherwise does not necessarily constitute or imply its endorsement, recommendation, or favoring by the United States Government or any agency thereof. The views and opinions of authors expressed herein do not necessarily state or reflect those of the United States Government or any agency thereof.

By acceptance of this arlicle, the publisher recognizes that the U.S. Government retains a nonexclusive, royalty-free license to publish or reproduce the published form of this contribution, or to allow others to do so, for U.S. Government purposes. 


\section{Hydrodynamic Instabilities in Inertial Confinement Fusion}

\section{Contents}

page

Foreword

Introduction and Survey of Instabilities

Linear analysis of Rayleigh-Taylor instability $\quad 5$

Superposed fluids with density discontinuity $\quad 6$

Effect of a continuous density gradient

Ablation-surface instability

Instability occurrence for opposed density and pressure gradients

Bubble rise in late-stage Rayleigh-Taylor instability

Saturation in intermediate-stage Rayleigh-Taylor instability

Saturation threshold

Haan saturation model

Instability calculations in ICF implosions

Linearized perturbation codes

Linear single-mode calculations in nonlinear radiation hydrodynamic code 32

Nonlinear multimode calculations 


\section{DISCLAIMER}

Portions of this document may be illegible in electronic image products. Images are produced from the best available original document. 


\section{Foreword}

This paper was prepared as a contribution to the Proceedings of the 45th Scottish Universities Summer School in Physics, held at the University of St. Andrews in August 1994. The School dealt with a range of topics in laser-plasma interactions, and was attended by about sixty graduate students and researchers from Europe and the United States. The paper was the basis for two lectures on the subject of hydrodynamic instabilities given at the School. The focus of the paper is on buoyancy-driven instabilities of the Rayleigh-Taylor type, which are commonly regarded as the most important kind of hydrodynamic instability in inertial-confinement-fusion implosions. The paper is intended to be pedagogical rather than research-oriented, and so is by no means a comprehensive review of work in this field. Rather, it is hoped that the student will find here a foundation on which to build an understanding of current research, and the experienced researcher will find a compilation of useful results.

The aim of the paper is to discuss the evolution of a single Rayleigh-Taylor-unstable mode, from its linear phase to its late-stage constant-velocity bubble growth, with a brief consideration of the saturation of linear growth. The influence of other modes is invoked only in the short-range sense (in wavenumber space) of the Haan saturation model. Owing to limitations of time in the lectures and of space in the Proceedings, the treatment of other instabilities such as Richtmyer-Meshkov and Kelvin-Helmholtz is necessarily very brief, and entirely inadequate as an introductory discussion. Likewise, there is no reference to the effect of convergent geometry, to long-range mode coupling, or to shape effects in threedimensional growth. Furthermore, there is no reference to the large body of experimental research related to hydrodynamic instabilities.

I would like to thank Chuck Cranfill, Steve Haan, and Brad Beck for their kindness in reviewing and commenting on preliminary drafts of the paper.

This work was supported by the United States Department of Energy under contract number W-7405-ENG-36. 


\title{
Hydrodynamic Instabilities in Inertial Confinement Fusion
}

\author{
Nelson M. Hoffman
}

\section{Introduction and Survey of Instabilities}

An ideal inertial-confinement-fusion (ICF) implosion is exactly spherically symmetric. If the implosion departs from spherical symmetry, the imploding capsule's performance is degraded in several ways. For example, the conversion of the imploding shell's kinetic energy to the fuel's internal energy becomes less efficient and the compression of the fuel to high density may be less extreme. In high-efficiency capsules ignited by a central highentropy bubble or "hotspot" (Lindl 1988), the surface area through which the hotspot loses energy by thermal conduction may be increased. Increased surface area also allows $\alpha$ particles created in fusion reactions to escape the hotspot, further hindering the hotspot's self-heating. In severe cases, asymmetry can lead to the breakup of the imploding shell (at larger spatial scales) or the creation of hydrodynamic turbulence (at smaller spatial scales). Turbulence in turn may have a number of deleterious effects, involving the turbulent transport of mass, momentum, and energy in ways that corrupt the highly organized evolving structure of the imploding capsule.

ICF implosions, whether real or ideal, are subject to a variety of hydrodynamic instabilities that amplify small departures from spherical symmetry. Instabilities can cause a disturbance to grow from an amplitude which may at first seem insignificant to a level that can seriously disrupt the flow, as described above. Instabilities do not themselves generate the initial asymmetric disturbance, or "seed", from which the final disruption grows. Instead, the seeds arise from limitations in our ability to fabricate perfectly spherical shells, to generate perfectly uniform laser beams, or to create perfectly symmetric thermal radiation fields in hohlraums. Small perturbations of a capsule's surface caused by the roughness of the material's crystal structure, or by machining marks from the fabrication process, are examples of instability seeds. Other examples include the interference pattern in a.focused laser spot, which can imprint disturbances on an initially smooth surface irradiated by the laser. Thus the seeds simply reflect the inevitable deviation of real-world experiments from the idealized constructs of theory. Instabilities then cause these seeds to grow to a size that may have serious consequences for an ICF implosion.

Hydrodynamic instabilities are straightforward consequences of the conservation equations of hydrodynamics. In their idealized form they are just solutions to these equations for specific initial and boundary conditions corresponding to somewhat simplified versions of real flow fields. For example, the Rayleigh-Taylor instability (Taylor 1950), which we shall encounter often in ICF in a generalized form, arises in the case of two initially motionless incompressible fluid layers of unequal density, where the denser fluid is supported atop the less dense fluid in a gravitational field. If the interface, or contact surface, between the layers is disturbed so as not to be exactly horizontal, then the Rayleigh-Taylor instability ensues. The interface disturbance, which is the initial seed in this case, grows until eventually bubbles of the less dense fluid ascend through the denser fluid while jets or "spikes" of the denser fluid plunge downward through the less dense fluid. The Rayleigh-Taylor in- 
stability is never encountered in this precise form in ICF, because gravitation plays no role in an ICF implosion; the time and space scales of ICF are simply too small. However, the accelerating and decelerating forces produced by pressure gradients acting on the shell of an ICF capsule are effectively analogous to gravity. Thus hydrodynamic phenomena arise which are for all practical purposes equivalent to the Rayleigh-Taylor instability, appropriately generalized. For example, in ICF we may encounter compressible flow fields which are converging or diverging, which do not necessarily have sharp boundaries separating fluids of different density, and in which the acceleration force is not necessarily constant in time.

A related instability is the ablation-surface instability, sometimes called "RayleighTaylor instability at an ablation surface," or the Bodner-Lindl instability (Bodner 1974, Lindl and Mead 1975). This occurs when intense radiation, either laser or thermal, heats a material interface and ablates it. The ablated material flows away from the interface, creating a high-pressure, low-density corona which accelerates the unablated material. The density decrease from unablated to ablated material corresponds to the contact surface between the two fluids in the incompressible Rayleigh-Taylor instability, while the pressure increase from unablated to ablated material gives an acceleration force. Two effects that are not present in the incompressible Rayleigh-Taylor instability act to reduce the growth rate in the ablation-surface instability: the ablation flow of material through the unstable region, and the smoothing of temperature perturbations by the radiation flux.

Other instabilities of particular consequence for ICF are the Richtmyer-Meshkov instability and the Kelvin-Helmholtz instability. The Richtmyer-Meshkov instability (Richtmyer 1960) occurs when a shock wave crosses the interface between two fluids of unequal density, traveling in a direction normal to the interface. This can be viewed as the limiting case of a Rayleigh-Taylor instability in which gravity acts for an infinitesimally short duration on the fluids, imparting an impulsive acceleration to the interface and generating fluid motions that persist even in the absence of gravity. The Kelvin-Helmholtz instability arises when the two fluids are initially in motion, and there is a variation across the interface of the velocity component parallel to the interface. Such a gradient in the parallel velocity, referred to as "velocity shear", is unstable if it is sufficiently severe, and leads to the creation of vortices which entrain the two fluids in a characteristic rotational motion. The KelvinHelmholtz instability (Kelvin 1910) can arise along the interface between the ascending bubbles and descending spikes of late-stage Rayleigh-Taylor instability, where the shear due to the differential motion of these structures can be significant. Other circumstances giving rise to Kelvin-Helmholtz instability include the case of a shock wave crossing an interface in a direction not precisely normal to the interface (Diamond et al. 1993). Shear is generated by the deviation from strict normality, so that both Kelvin-Helmholtz and Richtmyer-Meshkov instability can occur in this situation.

We shall see later that Rayleigh-Taylor instability and the related ablation-surface instability develop whenever the density and pressure gradients have opposite signs during an ICF capsule's implosion. This occurs primarily during two episodes of the implosion: ablation-surface instability arises at the outer surface of the capsule's shell during the initial inward acceleration of the shell, while Rayleigh-Taylor instability arises at the inner surface of the shell during the final deceleration of the shell by the low-density, high- 
pressure hotspot at the capsule's center. Between these two episodes, the shell is coasting at nearly constant velocity, and perturbations grow via the Richtmyer-Meshkov instability, induced by the shocks which emerge at the inside surface of the shell. If the shocks are not normal to the interface, Kelvin-Helmholtz instability may occur as well.

\section{A. Linear analysis of Rayleigh-Taylor Instability}

It is worthwhile to derive from basic principles the small-amplitude behavior of the Rayleigh-Taylor instability, both because we shall thereby discover some of the properties of the instability and because the exercise will serve as an example of the technique of linear perturbation analysis, widely used in instability studies. The discussion follows that of Chandrasekhar (1961). Our starting point is the system of equations describing the hydrodynamic motion of an ideal fluid (that is, a fluid in which there is no energy dissipation or heat exchange), known as the Euler equations:

$$
\begin{gathered}
\frac{\partial \rho}{\partial t}+\nabla \cdot(\rho \mathbf{v})=0 \\
\rho \frac{\partial \mathbf{v}}{\partial t}+\rho(\mathbf{v} \cdot \nabla) \mathbf{v}=-\nabla \mathrm{p}+\rho \mathbf{g} .
\end{gathered}
$$

Equation (A-1) is called the continuity equation and Eq. (A-2) is called the equation of motion or momentum equation. Here $\rho, \mathbf{v} \equiv \mathrm{v}_{\mathbf{x}} \hat{\mathbf{x}}+\mathrm{v}_{\mathbf{y}} \hat{\mathbf{y}}+\mathrm{v}_{\mathbf{z}} \hat{\mathbf{z}}$, and $p$ denote respectively the density, velocity, and pressure of the fluid. An external force, such as gravity, acting on the fluid is represented by $g \equiv g_{x} \hat{x}+g_{y} \hat{y}+g_{z} \hat{z}$. In the particular example of Rayleigh-Taylor instability we shall consider, the fluids meet at a horizontal interface and are initially at rest. We take the normal to the interface as the direction $\hat{\mathbf{z}}$, so that gravity acts along $\hat{\mathbf{z}}$. Since gravity acts downward, $g_{z}<0$ and $\mathbf{g}=-\left|g_{z}\right| \hat{\mathbf{z}}$. All physical quantities are initially uniform throughout both fluids, away from the interface.

To investigate the stability of hydrodynamic motion, we ask how the motion responds to a small fluctuation in the value of any of the flow variables appearing in the Euler equations. If the fluctuation grows in amplitude so that the flow never returns to its initial state, we say that the flow is unstable with respect to fluctuations of that type. Accordingly, we replace the variables in Eqs. (A-1) and (A-2) as follows:

$$
\begin{gathered}
\rho=\rho_{0}+\rho_{1}, \\
\mathbf{v}=\mathbf{v}_{0}+\mathbf{v}_{1}, \\
p=p_{0}+p_{1} .
\end{gathered}
$$

The quantities with subscripts " 0 " represent the unperturbed, or "zeroth-order" motion of the fluid, and thus must themselves satisfy Eqs. (A-1) and (A-2). The quantities with subscripts " 1 " represent a small perturbation about the zeroth-order quantities; that is, $\rho_{1}<<\rho_{0}, \mathbf{v}_{1}<<\mathbf{v}_{0}$, and $p_{1}<<p_{0}$. Substituting these expressions into Eqs. (A-1) and $(\mathrm{A}-2)$ gives

$$
\frac{\partial\left(\rho_{0}+\rho_{1}\right)}{\partial t}+\nabla \cdot\left[\left(\rho_{0}+\rho_{1}\right)\left(\mathbf{v}_{0}+\mathbf{v}_{1}\right)\right]=0
$$




$$
\begin{aligned}
& \left(\rho_{0}+\rho_{1}\right) \frac{\partial\left(\mathbf{v}_{0}+\mathbf{v}_{1}\right)}{\partial t}+\left(\rho_{0}+\rho_{1}\right)\left[\left(\mathbf{v}_{0}+\mathbf{v}_{1}\right) \cdot \nabla\right]\left(\mathbf{v}_{0}+\mathbf{v}_{1}\right)=-\nabla\left(p_{0}+p_{1}\right)+\left(\rho_{0}+\rho_{1}\right) \mathbf{g} \\
& \frac{\partial \rho_{0}}{\partial t}+\frac{\partial \rho_{1}}{\partial t}+\nabla \cdot\left(\rho_{0} \mathbf{v}_{0}+\rho_{1} \mathbf{v}_{0}+\rho_{0} \mathbf{v}_{1}+\rho_{1} \mathbf{v}_{1}\right)=0 \\
& \rho_{0} \frac{\partial \mathbf{v}_{0}}{\partial t}+\rho_{1} \frac{\partial \mathbf{v}_{0}}{\partial t}+\rho_{0} \frac{\partial \mathbf{v}_{1}}{\partial t}+\rho_{1} \frac{\partial \mathbf{v}_{1}}{\partial t}+ \\
& \rho_{0}\left(\mathbf{v}_{0} \cdot \nabla \mathbf{v}_{0}+\mathbf{v}_{1} \cdot \nabla \mathbf{v}_{0}+\mathbf{v}_{0} \cdot \nabla \mathbf{v}_{1}+\mathbf{v}_{1} \cdot \nabla \mathbf{v}_{1}\right)+\rho_{1}\left(\mathbf{v}_{0} \cdot \nabla \mathbf{v}_{0}+\mathbf{v}_{1} \cdot \nabla \mathbf{v}_{0}+\mathbf{v}_{0} \cdot \nabla \mathbf{v}_{1}+\mathbf{v}_{1} \cdot \nabla \mathbf{v}_{1}\right)= \\
& -\nabla p_{0}-\nabla p_{1}+\rho_{\circ} \mathbf{g}+\rho_{1} \mathbf{g}
\end{aligned}
$$

The fact that the zeroth-order quantities satisfy Eqs. (A-1) and (A-2) means

$$
\begin{gathered}
\frac{\partial \rho_{0}}{\partial t}+\nabla \cdot\left(\rho_{0} \mathbf{v}_{0}\right)=0 \\
\rho_{0} \frac{\partial \mathbf{v}_{0}}{\partial t}+\rho_{0}\left(\mathbf{v}_{0} \cdot \nabla\right) \mathbf{v}_{0}=-\nabla p_{0}+\rho_{0} \mathbf{g}
\end{gathered}
$$

We can subtract the zeroth-order equations Eqs. (A-5) and (A-6) from Eqs. (A-3) and (A-4). This amounts to dropping all terms in Eqs. (A-3) and (A-4) which contain no appearances of the subscript " 1 ". Furthermore, we can omit terms in Eqs. (A-3) and (A-4) which contain products of first-order quantities, since they are very small in comparison to terms which are linear in first-order quantities. This process of omission of quadratic quantities, by which we obtain a system of linear partial differential equations, is called linearization of the perturbed equations. Linearization is valid only if the perturbations are small. The result of linearizing and of subtracting the zeroth-order equations is that Eqs. (A-3) and (A-4) become

$$
\begin{array}{cc}
\frac{\partial \rho_{1}}{\partial t}+\nabla \cdot\left(\rho_{1} \mathbf{v}_{0}+\rho_{0} \mathbf{v}_{1}\right)=0, & (A-7) \\
\rho_{1} \frac{\partial \mathbf{v}_{0}}{\partial t}+\rho_{0} \frac{\partial \mathbf{v}_{1}}{\partial t}+\rho_{0}\left(\mathbf{v}_{1} \cdot \nabla \mathbf{v}_{0}+\mathbf{v}_{0} \cdot \nabla \mathbf{v}_{1}\right)+\rho_{1}\left(\mathbf{v}_{0} \cdot \nabla \mathbf{v}_{0}\right)=-\nabla p_{1}+\rho_{1} \mathbf{g} . & (A-8)
\end{array}
$$

\section{Superposed fiuids with density discontinuity}

We now restrict our attention to the Rayleigh-Taylor instability in particular. For the problem as it was posed earlier, the fluids are initially at rest. This means that $\mathbf{v}_{0}=0$, so that Eqs. (A-7) and (A-8) become

$$
\frac{\partial \rho_{1}}{\partial t}+\nabla \cdot\left(\rho_{0} \mathbf{v}_{1}\right)=0
$$




$$
\rho_{0} \frac{\partial \mathbf{v}_{1}}{\partial t}=-\nabla p_{1}+\rho_{1} \mathrm{~g}
$$

We now appeal to the fact that, for many situations of interest in ICF, unstable flow occurs at velocities much smaller than the local sound speed. This has the effect that accelerations in the flow are not strong enough to change the density of a fluid element significantly, so the fluid moves without compressing or expanding. In such a situation we call the flow incompressible. Provided that we are well away from shock waves or centers of convergence, the assumption of incompressible flow is often valid. To say that fluid elements move without changing density is to say that the Lagrangian total derivative (also called the total substantive derivative) of density is zero, that is,

$$
\frac{d \rho}{d t}=\frac{\partial \rho}{\partial t}+\mathbf{v} \cdot \nabla \rho=0
$$

Applying this equation to our instability analysis, we substitute the perturbed expressions $\rho=\rho_{0}+\rho_{1}$ and $\mathbf{v}=\mathbf{v}_{0}+\mathbf{v}_{1}$ into Eq. (A-11), and recall that both $\mathbf{v}_{0}$ and the time derivative of $\rho_{0}$ vanish, since they describe the static initial state. We also linearize the result, dropping nonlinear terms in the first-order quantities, as before. The result is that Eq. (A-11) becomes

$$
\frac{\partial \rho_{1}}{\partial t}+\mathbf{v}_{1} \cdot \nabla \rho_{0}=0
$$

Comparing this equation to Eq. (A-9), which we write in expanded form as

$$
\frac{\partial \rho_{1}}{\partial t}+\rho_{0} \nabla \cdot \mathbf{v}_{1}+\mathbf{v}_{1} \cdot \nabla \rho_{0}=0
$$

we see that subtracting Eq. (A-12) from Eq. (A-13) yields

$$
\nabla \cdot \mathbf{v}_{1}=0
$$

This is a consequence of the assumption of incompressible flow. We can use either Eq. (A-12) or Eq. (A-14) to replace the linearized continuity equation Eq. (A-9) under this assumption.

To proceed, we write out the vector equations (A-10) and (A-12) in component form. The linearized momentum equation (A-10) becomes

$$
\begin{aligned}
& \rho_{0} \frac{\partial v_{1 x}}{\partial t}=-\frac{\partial p_{1}}{\partial x}+\rho_{1} g_{x}, \\
& \rho_{0} \frac{\partial v_{1 y}}{\partial t}=-\frac{\partial p_{1}}{\partial y}+\rho_{1} g_{y}, \\
& \rho_{0} \frac{\partial v_{1 z}}{\partial t}=-\frac{\partial p_{1}}{\partial z}+\rho_{1} g_{z},
\end{aligned}
$$


while the linearized incompressible continuity equation (A-12) becomes

$$
\frac{\partial \rho_{1}}{\partial t}+v_{1 x} \frac{\partial \rho_{0}}{\partial x}+v_{1 y} \frac{\partial \rho_{0}}{\partial y}+v_{1 z} \frac{\partial \rho_{0}}{\partial z}=0 .
$$

Because gravity acts only in the $\hat{\mathbf{z}}$ direction, $g_{x}=g_{y}=0$. Furthermore, since $\rho_{0}$ is uniform throughout each medium, with its only variation occurring across the horizontal interface, we have $\partial \rho_{0} / \partial x=\partial \rho_{0} / \partial y=0$, while $\partial \rho_{0} / \partial z$ is non-zero, but only at the interface. Thus the linearized incompressible component equations may be written, using $g=\left|g_{z}\right|=-g_{z}$,

$$
\begin{gathered}
\rho_{0} \frac{\partial v_{1 x}}{\partial t}=-\frac{\partial p_{1}}{\partial x}, \\
\rho_{0} \frac{\partial v_{1 y}}{\partial t}=-\frac{\partial p_{1}}{\partial y}, \\
\rho_{0} \frac{\partial v_{1 z}}{\partial t}=-\frac{\partial p_{1}}{\partial z}-\rho_{1} g, \\
\frac{\partial \rho_{1}}{\partial t}+v_{1 z} \frac{\partial \rho_{0}}{\partial z}=0 .
\end{gathered}
$$

It will also be useful to have Eq. (A-14), which expresses the nondivergence of the firstorder flow, in component form:

$$
\frac{\partial v_{1 x}}{\partial x}+\frac{\partial v_{1 y}}{\partial y}+\frac{\partial v_{1 z}}{\partial z}=0 .
$$

The next step in our analysis is to carry out a Fourier transformation of the system of equations (A-15) - (A-19). This is a powerful technique for the solution of differential equations, because of a useful property of Fourier transforms: if $F[f(t)]$ is the Fourier transform of the function $f(t)$ with respect to the independent variable $t$, then the Fourier transform of the derivative $d f / d t$ is

$$
F[d f / d t]=i s F[f(t)],
$$

where $s$ is the transform variable. Thus a differential operator acting on a physical quantity becomes simply a product of the corresponding transform variable and the Fourier transform of that quantity. Accordingly we define the following two-dimensional Fourier transforms with respect to $x$ and $y$ :

$$
\begin{gathered}
V_{1 x}\left(k_{x}, k_{y}, z, t\right)=F_{x y}\left[v_{1 x}(x, y, z, t)\right] \\
\equiv \frac{1}{2 \pi} \int_{-\infty}^{+\infty} d x \int_{-\infty}^{+\infty} d y v_{1 x}(x, y, z, t) e^{i\left(k_{x} x+k_{y} y\right)}, \\
V_{1 y}\left(k_{x}, k_{y}, z, t\right)=F_{x y}\left[v_{1 y}(x, y, z, t)\right] \\
V_{1 z}\left(k_{x}, k_{y}, z, t\right)=F_{x y}\left[v_{1 z}(x, y, z, t)\right]
\end{gathered}
$$




$$
\begin{aligned}
& P\left(k_{x}, k_{y}, z, t\right)=F_{x y}\left[p_{1}(x, y, z, t)\right], \\
& R\left(k_{x}, k_{y}, z, t\right)=F_{x y}\left[\rho_{1}(x, y, z, t)\right] .
\end{aligned}
$$

We do not transform with respect to $z$, because the $\hat{\mathbf{z}}$ direction does not share the symmetry of the other two directions; the linearized component equations (A-15)-(A-19) are invariant under the interchange of $x$ and $y$, and it will turn out that solutions are waves in the $(x, y)$ plane. Furthermore, zeroth-order quantities such as $\rho_{0}$ are not functions of $x$ and $y$, which simplifies the Fourier integrals. The transform variables $k_{x}$ and $k_{y}$ are called the $\hat{\mathbf{x}}$ and $\hat{\mathbf{y}}$ components, respectively, of the wavevector $\mathbf{k}$, whose magnitude $k=\sqrt{k_{x}^{2}+k_{y}^{2}}$ is called the wavenumber. Corresponding to the wavenumber is a wavelength $\lambda=2 \pi / k$.

Additionally, we shall seek solutions whose time dependence is proportional to $e^{\gamma t}$. This is a standard procedure when Fourier transforming differential equations. If, for example, we suppose that

$$
V_{1 z}\left(k_{x}, k_{y}, z, t\right)=\tilde{V}_{1 z}\left(k_{x}, k_{y}, z\right) e^{\gamma t}
$$

then

$$
\frac{\partial V_{1 z}}{\partial t}=\gamma \tilde{V}_{1 z} e^{\gamma t}=\gamma V_{1 z}
$$

Thus, again, a derivative can be replaced by a product. The variable $\gamma$ is called the frequency.

Performing the Fourier transforms of the component equations (A-15)-(A-19), and making the assumption that the time dependence of the solution is given by $e^{\gamma t}$, where $\gamma$ may be a function of $k_{x}$ and $k_{y}$, results in

$$
\begin{gathered}
\gamma \rho_{0} V_{1 x}=-i k_{x} P, \\
\gamma \rho_{0} V_{1 y}=-i k_{y} P, \\
\gamma \rho_{0} V_{1 z}=-\frac{\partial P}{\partial z}-g R, \\
\gamma R+V_{1 z} \frac{\partial \rho_{0}}{\partial z}=0, \\
i k_{x} V_{1 x}+i k_{y} V_{1 y}+\frac{\partial V_{1 z}}{\partial z}=0 .
\end{gathered}
$$

The solution of the equations (A-20) - (A-24) is now straightforward. Multiply Eq. (A-20) by $i k_{x}$ and multiply Eq. (A-21) by $i k_{y}$ :

$$
\begin{aligned}
& i \gamma \rho_{0} k_{x} V_{1 x}=k_{x}^{2} P \\
& i \gamma \rho_{0} k_{y} V_{1 y}=k_{y}^{2} P .
\end{aligned}
$$

Add these equations:

$$
i \gamma \rho_{0}\left(k_{x} V_{1 x}+k_{y} V_{1 y}\right)=\left(k_{x}{ }^{2}+k_{y}{ }^{2}\right) P
$$


From Eq. (A-24), solve for $k_{x} V_{1 x}+k_{y} V_{1 y}=i \partial V_{1 z} / \partial z$ and substitute in Eq. (A-25):

$$
-\gamma \rho_{0} \frac{\partial V_{1 z}}{\partial z}=k^{2} P
$$

using $k^{2}=k_{x}^{2}+k_{y}^{2}$, the square of the wavenumber. Next we eliminate $R$ between Eqs. (A-22) and (A-23). Equation (A-23) implies

$$
R=-\frac{1}{\gamma} V_{1 z} \frac{\partial \rho_{0}}{\partial z}
$$

which we may substitute into Eq. (A-22) to obtain

$$
\frac{\partial P}{\partial z}=-\gamma \rho_{0} V_{1 z}+\frac{g}{\gamma} V_{1 z} \frac{\partial \rho_{0}}{\partial z} .
$$

Finally, solving for $P$ from Eq. (A-26) and inserting here we find

$$
\frac{\partial}{\partial z}\left(\rho_{0} \frac{\partial V_{1 z}}{\partial z}\right)=k^{2} \rho_{0} V_{1 z}\left(1-\frac{g}{\gamma^{2} \rho_{0}} \frac{\partial \rho_{0}}{\partial z}\right)
$$

Equation (A-27) does not have solutions for arbitrary values of $\gamma$, once $k, g$, and $\rho_{0}(z)$ are defined. Solutions exist only for a particular value of $\gamma$, called the characteristic value or eigenvalue. Equation (A-27) is called an eigenvalue equation, and its solutions $V_{1 z}$ are called eigenfunctions.

Since we are considering fluids of uniform density, $\rho_{0}$ is constant everywhere except at the interface. Therefore, away from the interface, $\partial \rho_{0} / \partial z$ vanishes and $\rho_{0}$ may be canceled from Eq. (A-27), leaving

$$
\frac{\partial^{2} V_{1 z}}{\partial z^{2}}=k^{2} V_{1 z}
$$

The general solution to this equation is

$$
V_{1 z}=A e^{+k z}+B e^{-k z}
$$

The vertical velocity should vanish at large distances from the interface, and so we choose a solution with $A \neq 0, B=0$ for $z<0$ and with $A=0, B \neq 0$ for $z>0$. $V_{1 z}$ must be continuous across the interface since a velocity discontinuity would require an infinite acceleration and therefore an infinite force. Accordingly we select

$$
V_{1 z}=\left\{\begin{array}{l}
W e^{+k z}, z<0 \\
W e^{-k z}, z>0
\end{array}\right\},
$$

where $W=V_{1 z}(z=0)$ is the value at the interface.

The derivative $\partial V_{1 z} / \partial z$ is not continuous, however. It has the value $k W$ immediately below the interface and $-k W$ immediately above. Equation (A-27) expresses the relationship between the discontinuity in $\partial V_{1 z} / \partial z$ and the discontinuity in density: We can 
use this relationship to derive a boundary condition at the interface and determine the frequency eigenvalue $\gamma$ in terms of the gravity $g$, the wavenumber $k$, and the density jump.

To do so, we integrate Eq. (A-27) over an infinitesimal element of $z$ that includes the interface $z=0$. The derivative of a quantity, when integrated, then gives simply the change in the value of that quantity across the interface. Thus the left-hand side of Eq. (A-27) integrates to

$$
\begin{aligned}
\int_{-\epsilon}^{\epsilon} \frac{\partial}{\partial z}\left(\rho_{0} \frac{\partial V_{1 z}}{\partial z}\right) d z & =\left.\rho_{0} \frac{\partial V_{1 z}}{\partial z}\right|_{-\epsilon} ^{\epsilon}=-\rho_{0}(z>0) k W-\rho_{0}(z<0) k W \\
& =-k W\left(\rho_{\text {above }}+\rho_{\text {below }}\right) \equiv I_{1},
\end{aligned}
$$

where $\rho_{a b o v e} \equiv \rho_{0}(z>0)$ is the density in the upper fluid and $\rho_{\text {below }} \equiv \rho_{0}(z<0)$ is the density in the lower fluid. The first term on the right-hand side of Eq. (A-27) gives, upon integration,

$$
\int_{-\epsilon}^{\epsilon} k^{2} \rho_{0} V_{1 z} d z=k^{2} W\left(\rho_{a b o v e} \epsilon+\rho_{b e l o w} \epsilon\right) \equiv I_{2}
$$

The second term on the right-hand side of Eq. (A-27) gives

$$
\begin{gathered}
-\int_{-\epsilon}^{\epsilon} k^{2} \rho_{0} V_{1 z} \frac{g}{\gamma^{2} \rho_{0}} \frac{\partial \rho_{0}}{\partial z} d z=-\frac{k^{2} W g}{\gamma^{2}} \int_{-\epsilon}^{\epsilon} \frac{\partial \rho_{0}}{\partial z} d z=-\left.\frac{k^{2} W g}{\gamma^{2}} \rho_{0}\right|_{-\epsilon} ^{\epsilon} \\
=-\frac{k^{2} W g}{\gamma^{2}}\left(\rho_{\text {above }}-\rho_{\text {below }}\right) \equiv I_{3} .
\end{gathered}
$$

In the limit that $\epsilon$ goes to zero, $I_{2}$ vanishes, because it is proportional to $\epsilon$. On the other hand, $I_{1}$ and $I_{3}$ are finite; they are, in effect, integrals of delta functions. Thus we must have $I_{1}=I_{3}$ or

$$
-k W\left(\rho_{a b o v e}+\rho_{\text {below }}\right)=-\frac{k^{2} W g}{\gamma^{2}}\left(\rho_{\text {above }}-\rho_{\text {below }}\right) .
$$

Solving for $\gamma$, we obtain

$$
\gamma^{2}=k g \frac{\left(\rho_{\text {above }}-\rho_{\text {below }}\right)}{\left(\rho_{\text {above }}+\rho_{\text {below }}\right)} .
$$

Define a dimensionless number $A$, called the Atwood number:

$$
A \equiv \frac{\left(\rho_{\text {above }}-\rho_{\text {below }}\right)}{\left(\rho_{\text {above }}+\rho_{\text {below }}\right)} .
$$

Then $\gamma^{2}=k g A$. Since solutions depend on time as $e^{\gamma t}$, we have, for example,

$$
V_{1 z}=\left\{\begin{array}{l}
\tilde{W}\left(k_{x}, k_{y}\right) e^{+k z} e^{\gamma t}, z<0 \\
\tilde{W}\left(k_{x}, k_{y}\right) e^{-k z} e^{\gamma t}, z>0
\end{array}\right\} .
$$


If $\rho_{a b a v e}>\rho_{b e l o w}$, then $A$ is positive, the interface is unstable, and the perturbation grows exponentially with growth rate $\gamma=\sqrt{k g A}$. If, on the other hand, $\rho_{\text {above }}<\rho_{\text {below }}$, then $A$ is negative, $\gamma$ is imaginary, and the interface oscillates with frequency $\operatorname{Im}(\gamma)=\sqrt{k g A}$.

\section{Effect of a continuous density gradient}

In actual ICF situations, the interface between two materials is never truly a discontinuity. Instead, the density varies continuously with position, although sometimes the gradient can be rather steep. Often, however, the density varies continuously with a length scale comparable to perturbation wavelengths of interest. To find the effect of a continuous density variation on the growth rate of the instability, we need to repeat the above analysis with a smooth variation of $\rho_{0}(z)$.

A treatment of this problem was carried out by LeLevier et al. (1955) for a model density profile defined by

$$
\rho_{0}(z)=\left\{\begin{array}{c}
\rho_{\text {below }}+\frac{\Delta \rho}{2} e^{+K z}, z<0 \\
\rho_{\text {above }}-\frac{\Delta \rho}{2} e^{-K z}, z>0
\end{array}\right\},
$$

where $\Delta \rho \equiv \rho_{\text {above }}-\rho_{\text {below }}$. Their starting point is the system of linearized incompressible component equations (A-15) - (A-18). If we assume sinusoidal variations in $x$ and $y$ for all variables, we can omit Eqs. (A-15) and (A-16), leaving just Eqs. (A-17) and (A-18):

$$
\begin{gathered}
\rho_{0} \frac{\partial v_{1 z}}{\partial t}=-\frac{\partial p_{1}}{\partial z}-\rho_{1} g \\
\frac{\partial \rho_{1}}{\partial t}+v_{1 z} \frac{\partial \rho_{0}}{\partial z}=0 .
\end{gathered}
$$

Further, assume that $v_{1 z}$ is given by

$$
v_{1 z}(x, z, t)=\left\{\begin{array}{l}
e^{+k z} \cos k x f(t), z<0 \\
e^{-k z} \cos k x f(t), z>0
\end{array}\right\} .
$$

This is an approximation, because this form for the eigenfunction $v_{1 z}$ actually corresponds to the solution Eq. (A-31) for the case of discontinuous density. All the same, it leads to a simple expression for the instability growth rate which is useful and illustrates qualitatively the effect of a continuous density gradient. Mikaelian (1986) calculated accurate numerical eigenfunctions and the corresponding growth rates, for the density profile given by Eq. (A32 ), and found that the approximate eigenfunction gives quite good results.

So we proceed to integrate Eq. (A-34) over time to get an expression for $\rho_{1}$ :

$$
\rho_{1}(x, z, t)=-\frac{\partial \rho_{0}}{\partial z} \int_{0}^{t} v_{1 z} d t^{\prime},
$$


where we assume that the initial density perturbation is zero and the zeroth-order density $\rho_{0}(z)$ is static. This can be inserted into Eq. (A-33), which when rearranged becomes

$$
\frac{\partial p_{1}}{\partial z}=-\rho_{0} \frac{\partial v_{1 z}}{\partial t}+g \frac{\partial \rho_{0}}{\partial z} \int_{0}^{t} v_{1 z} d t^{\prime}
$$

Using expressions (A-32) and (A-35) for $\rho_{0}(z)$ and $v_{1 z}$, respectively, this becomes

$$
\frac{\partial p_{1}}{\partial z}=-\cos k x\left\{\begin{array}{l}
{\left[\rho_{\text {below }}+\frac{\Delta \rho}{2} e^{+K z}\right] e^{+k z} \dot{f}+g K \frac{\Delta \rho}{2} e^{+(K+k) z} \int_{0}^{t} f\left(t^{\prime}\right) d t^{\prime}, z<0} \\
{\left[\rho_{\text {above }}-\frac{\Delta \rho}{2} e^{-K z}\right] e^{-k z} \dot{f}+g K \frac{\Delta \rho}{2} e^{-(K+k) z} \int_{0}^{t} f\left(t^{\prime}\right) d t^{\prime}, z>0}
\end{array}\right\} .
$$

This expression for the derivative of the first-order pressure may be integrated from $z=-\infty$ to $z=0$ to obtain an expression for $p_{1}(z=0)$, since $p_{1}(z=-\infty)$ vanishes. Likewise, integrating from $z=0$ to $z=+\infty$ gives another expression for $p_{1}(z=0)$. These expressions must be equal, since $p_{1}$ is continuous at $z=0$ for a continuous density profile, unlike the case in our earlier analysis for discontinuous density. Carrying out the integration gives

$$
p_{1}(z=0)=\int_{-\infty}^{0} \frac{\partial p_{1}}{\partial z} d z=-\int_{0}^{\infty} \frac{\partial p_{1}}{\partial z} d z
$$

Thus

$$
\begin{aligned}
& -\dot{f}\left[\frac{\rho_{\text {below }}}{k}+\frac{\Delta \rho}{2} \frac{1}{K+k}\right]+\left(\int_{0}^{t} f d t^{\prime}\right) \frac{g \Delta \rho}{2} \frac{K}{K+k} \\
& =\dot{f}\left[\frac{\rho_{a b o v e}}{k}-\frac{\Delta \rho}{2} \frac{1}{K+k}\right]-\left(\int_{0}^{t} f d t^{\prime}\right) \frac{g \Delta \rho}{2} \frac{K}{K+k}
\end{aligned}
$$

or

$$
\dot{f}\left(\frac{\rho_{\text {above }}+\rho_{\text {below }}}{k}\right)-g \Delta \rho \frac{K}{K+k}\left(\int_{0}^{t} f d t^{\prime}\right)=0 .
$$

Differentiating and rearranging coefficients gives

$$
\ddot{f}-g \frac{k K}{K+k}\left(\frac{\rho_{a b o v e}-\rho_{\text {below }}}{\rho_{\text {above }}+\rho_{\text {below }}}\right) f=0 .
$$

The solution is an exponential with growth rate

$$
\gamma=\sqrt{\frac{g k K}{K+k} A}
$$

where $A$ is the Atwood number. If we define a gradient length $L \equiv 1 / K$, we can express the growth rate as

$$
\gamma=\sqrt{\frac{g k A}{1+k L}}
$$


which is a commonly encountered form.

It is clear from this expression that the effect of the density gradient is always to reduce the growth rate of the instability. For perturbation wavelengths much shorter than $L$, or, equivalently, for long gentle gradients, we have $k L>>1$, so $\gamma \rightarrow \sqrt{g A / L} \equiv$ $\sqrt{g K A}$, independent of wavelength. The gradient therefore limits the growth rate for shortwavelength perturbations to the growth rate of a mode whose wavenumber equals $K$, the inverse lengthscale of the gradient. On the other hand, for perturbation wavelengths much longer than $L$, or, equivalently, for steep gradients, we have $k L<<1$, so the growth rate $\gamma \simeq \sqrt{g k A}$, unaffected by the density gradient.

\section{B. Ablation-Surface Instability}

The ablation-surface instability occurs when a material layer is rapidly heated by some energy-deposition process and ablates. If the spatial extent of the energy-deposition region is small with respect to the depth of the layer, then a high-pressure low-density region forms adjacent to the layer, which accelerates the layer. The low-density region is composed of heated ablating material expanding away from the layer's surface. The acceleration of the high-density layer by the low-density ablated material is analogous to the support of a high-density fluid by a low-density fluid in a gravitational field, so an instability arises. This ablation-surface instability is much like the classical Rayleigh-Taylor instability, just discussed, but differs because of the flow of material out of the high-density layer, across the ablation surface, and into the low-density ablated region. Furthermore, gravity plays no role.

If we approximate the energy-deposition region as a discontinuity, we can make a rough estimate (following Gamaly 1993) of the effect of ablation on the growth of perturbations by repeating the Rayleigh-Taylor analysis with a simple change: because of the ablation flow, we permit a velocity discontinuity at the interface as well as a density discontinuity. This means that the zeroth-order state is not static, so that we cannot set $\mathbf{v}_{0}=0$ in our linear perturbation analysis.

We consider a reference frame moving with the layer. In this frame, the layer is at rest, and the ablating material moves in the $-\hat{\mathbf{z}}$ direction with velocity $v_{a b l}$. Thus

$$
\mathbf{v}_{0}=\left\{\begin{array}{r}
-v_{a b l \hat{\mathbf{z}}, z<0} \\
0, z>0
\end{array}\right\} .
$$

Rewriting Eq. (A-8), omitting gravity, and keeping terms containing $\mathbf{v}_{0}$ gives

$$
\rho_{0} \frac{\partial \mathbf{v}_{1}}{\partial t}+\rho_{1}\left(\frac{\partial \mathbf{v}_{0}}{\partial t}+\mathbf{v}_{0} \cdot \nabla \mathbf{v}_{0}\right)+\rho_{0}\left(\mathbf{v}_{1} \cdot \nabla \mathbf{v}_{0}+\mathbf{v}_{0} \cdot \nabla \mathbf{v}_{1}\right)=-\nabla p_{1}
$$

Rewriting Eq. (A-6) and omitting gravity gives

$$
\frac{\partial \mathbf{v}_{0}}{\partial t}+\mathbf{v}_{0} \cdot \nabla \mathbf{v}_{0}=-\frac{1}{\rho_{0}} \nabla p_{0} .
$$

We may substitute this expression for the total Lagrangian derivative of $\mathbf{v}_{0}$ into Eq. (B-1) and rearrange terms to obtain

$$
\rho_{0}\left(\frac{\partial \mathbf{v}_{1}}{\partial t}+\mathbf{v}_{1} \cdot \nabla \mathbf{v}_{0}+\mathbf{v}_{0} \cdot \nabla \mathbf{v}_{1}\right)=\frac{\rho_{1}}{\rho_{0}} \nabla p_{0}-\nabla p_{1}
$$


Just as in the classical Rayleigh-Taylor analysis, we take the two-dimensional Fourier transform of the $z$-component of Eq. (B-3), assuming a time dependence like $e^{\gamma t}$. This leads to

$$
\rho_{0}\left(\gamma V_{1 z}+V_{1 z} \frac{\partial v_{0 z}}{\partial z}+v_{0 z} \frac{\partial V_{1 z}}{\partial z}\right)=\frac{R}{\rho_{0}} \frac{\partial p_{0}}{\partial z}-\frac{\partial P}{\partial z},
$$

where, as before, $V_{1 z}=F\left[v_{1 z}\right], R=F\left[\rho_{1}\right]$, and $P=F\left[p_{1}\right]$.

We define an acceleration $g_{0} \equiv-\left(1 / \rho_{0}\right) \partial p_{0} / \partial z$, and we use Eq. (A-23) to eliminate $R=-(1 / \gamma) V_{1 z} \partial \rho_{0} / \partial z$ in Eq. (B-4). Then solving Eq. (B-4) for $\partial P / \partial z$ results in

$$
\frac{\partial P}{\partial z}=-\gamma \rho_{0} V_{1 z}+\frac{g_{0}}{\gamma} V_{1 z} \frac{\partial \rho_{0}}{\partial z}-\rho_{0} V_{1 z} \frac{\partial v_{0 z}}{\partial z}-\rho_{0} v_{0 z} \frac{\partial V_{1 z}}{\partial z} .
$$

Finally, we use Eq. (A-26) to eliminate $P$ in terms of $\partial V_{1 z} / \partial z$, and find upon multiplying by $-k^{2} / \gamma$

$$
\frac{\partial}{\partial z}\left(\rho_{0} \frac{\partial V_{1 z}}{\partial z}\right)=k^{2} \rho_{0} V_{1 z}-g_{0} \frac{k^{2}}{\gamma^{2}} V_{1 z} \frac{\partial \rho_{0}}{\partial z}+\frac{k^{2}}{\gamma} \rho_{0} V_{1 z} \frac{\partial v_{0 z}}{\partial z}+\frac{k^{2}}{\gamma} \rho_{0} v_{0 z} \frac{\partial V_{1 z}}{\partial z} . \quad(B-6)
$$

Equation (B-6) is analogous to Eq. (A-27) in the classical Rayleigh-Taylor analysis, but contains two additional terms on the right-hand side, proportional to the zeroth-order velocity and its $z$-gradient. Again, we use this equation to derive a jump condition at the interface by integrating it over an infinitesimal element $-\epsilon \leq z \leq \epsilon$, which includes the interface. We shall find as usual that only the terms in Eq. (B-6) which are delta functions produce any finite contribution to the integral in the limit that $\epsilon \rightarrow 0$. These are the second and third terms on the right-hand side, and the term on the left-hand side; $\rho_{0}, v_{0 z}$, and $\partial V_{1 z} / \partial z$ are discontinuous at the interface, so their $z$-derivatives are delta functions. However, $\partial V_{1 z} / \partial z$ itself is not a delta function, so the fourth term on the righthand side produces a vanishing integral. So does the first term on the right-hand side, as we saw in Eq. (A-29) in the classical Rayleigh-Taylor analysis.

Integrating Eq. (B-6) requires evaluating only one new term, since two of the nonvanishing terms were integrated earlier, as $I_{1}$ in Eq. (A-28) and $I_{3}$ in Eq. (A-30). The new integral is

$$
\int_{-\epsilon}^{\epsilon} \frac{k^{2}}{\gamma} \rho_{0} V_{1 z} \frac{\partial v_{0 z}}{\partial z} d z=k^{2} \frac{W}{\gamma} \int_{-\epsilon}^{\epsilon} \rho_{0} \frac{\partial v_{0 z}}{\partial z} d z \equiv I_{4}
$$

where $W$ is the value of $V_{1 z}$ at $z=0$. The integral is not so straightforward to evaluate as those encountered earlier, since the integrand is the product of a step function and a delta function. However, let us suppose that $\rho_{0}$ and $v_{0}$ vary linearly over the infinitesimal region $-\epsilon \leq z \leq \epsilon$, so that as $\epsilon \rightarrow 0$ they approach step functions and $\partial v_{0 z} / \partial z$ approaches a delta function. Then the integral is trivial, with the result that

$$
I_{4}=k^{2} \frac{W}{\gamma} \frac{\rho_{u n}+\rho_{a b l}}{2} v_{a b l},
$$

where $\rho_{u n}$ and $\rho_{a b l}$ are the densities in the unablated layer and in the ablated region, respectively. 
Thus the result of integrating Eq. (B-6) is

$$
I_{1}=I_{3}+I_{4}
$$

or

$$
-k W\left(\rho_{u n}+\rho_{a b l}\right)=-\frac{k^{2} W g_{0}}{\gamma^{2}}\left(\rho_{u n}-\rho_{a b l}\right)+\frac{k^{2} W}{\gamma} \frac{\rho_{u n}+\rho_{a b l}}{2} v_{a b l},
$$

which can be simplified as

$$
\gamma^{2}+\frac{k v_{a b l}}{2} \gamma-k g_{0} A=0
$$

where

$$
A \equiv \frac{\left(\rho_{u n}-\rho_{a b l}\right)}{\left(\rho_{u n}+\rho_{a b l}\right)} .
$$

The solution to the quadratic equation (B-7) for $\gamma$ is

$$
\gamma=-\frac{k v_{a b l}}{4} \pm \sqrt{\left(\frac{k v_{a b l}}{4}\right)^{2}+k g_{0} A} .
$$

The positive root may be written, when $\sqrt{k g_{0} A}>>k v_{a b l} / 4$,

$$
\gamma=\sqrt{k g_{0} A}-\frac{k v_{a b l}}{4}+\frac{1}{2 \sqrt{k g_{0} A}}\left(\frac{k v_{a b l}}{4}\right)^{2}-\ldots
$$

The effect of ablation is thus to reduce the growth rate of the instability.

Although this expression is only approximate, having been derived under some rather severe restrictions (no spatial extent of the region of acceleration, no modification of the continuity equation for finite zeroth-order velocity, no heating or energy exchange), it nevertheless resembles relations obtained from more accurate treatments. For example, the Takabe relation (Takabe et al. 1985)

$$
\gamma=\alpha \sqrt{k g_{0}}-\beta k v_{a}
$$

is found to describe detailed numerical solutions of a linear perturbation analysis of ablation-surface instability that includes heating and energy exchange in the flow. The analysis results in a system of five coupled ordinary differential equations for first-order variations in five quantities: density, normal velocity, tangential velocity, temperature, and normal heat flux. In general, the solutions are well fit using $\alpha=0.9$ and $3<\beta<4$. In Eq. (B-9), the ablation velocity $v_{a}$ denotes the mass ablation rate divided by the density at the ablation surface, whereas $v_{a b l}$ in Eq. (B-8) represents the terminal velocity reached by ablating material far from the ablation surface. We expect $v_{a b l}>>v_{a}$, which accounts in part for the different coefficients of $k v_{a b l}$ and $k v_{a}$ in eqs. (B-8) and (B-9).

The effect of ablation in reducing the growth rate of the instability is crucial to the success of ICF implosions. Equation (B-9) demonstrates that the growth of high wavenumbers (short wavelengths) is reduced more effectively than the growth of low wavenumbers. 
In fact; there is a "cutoff" wavenumber $k_{c u t}=(\alpha / \beta)^{2}\left(g_{0} / v_{a}^{2}\right)$ for which the growth rate is zero, and above which modes are stable.

Instability occurrence for opposed density and pressure gradients

Earlier it was pointed out that Rayleigh-Taylor-like instabilities arise wherever the density gradient and the pressure gradient have opposite signs in an imploding capsule. To see this, factor Eq. (B-6):

$$
\frac{\partial}{\partial z}\left(\rho_{0} \frac{\partial V_{1 z}}{\partial z}\right)=k^{2} \rho_{0} V_{1 z}\left(1-\frac{g_{0}}{\gamma^{2} \rho_{0}} \frac{\partial \rho_{0}}{\partial z}+\frac{1}{\gamma} \frac{\partial v_{0 z}}{\partial z}+\frac{v_{0 z}}{\gamma V_{1 z}} \frac{\partial V_{1 z}}{\partial z}\right)
$$

Recall that $g_{0}$ was merely shorthand for $-\left(1 / \rho_{0}\right) \partial p_{0} / \partial z$, so

$$
\frac{\partial}{\partial z}\left(\rho_{0} \frac{\partial V_{1 z}}{\partial z}\right)=k^{2} \rho_{0} V_{1 z}\left(1+\frac{1}{\gamma^{2}} \frac{1}{\rho_{0}^{2}} \frac{\partial p_{0}}{\partial z} \frac{\partial \rho_{0}}{\partial z}+\frac{1}{\gamma} \frac{\partial v_{0 z}}{\partial z}+\frac{v_{0 z}}{\gamma V_{1 z}} \frac{\partial V_{1 z}}{\partial z}\right)
$$

The terms in $v_{0}$ are stabilizing, as we have seen. The second term on the right-hand side drives the instability if its sign is negative, which will be the case if $\partial p_{0} / \partial z$ and $\partial \rho_{0} / \partial z$ do not have the same sign.

\section{Bubble rise in late-stage Rayleigh-Taylor instability}

The amplitude of a sinusoidal perturbation increases exponentially with time in the early stage of Rayleigh-Taylor instability, as we saw earlier in the linear analysis. Eventually the growth rate decreases, when the amplitude becomes about $10 \%$ of the wavelength $\lambda=2 \pi / k$. At this point, higher harmonics of the original sinusoid appear. The perturbed interface is then no longer sinusoidal, but assumes a "bubble-and-spike" configuration, in which rising, broader bubbles alternate with falling, narrower spikes. The relative width of bubbles and spikes depends on the density ratio of the two fluids, or, equivalently, on the Atwood number $A$. When $A \simeq 1$, the bubbles are much broader than the spikes. But when $A \simeq 0$, that is, the fluids have nearly the same density, there is little distinction between the behavior of bubbles and spikes, and they have nearly the same width.

Eventually the flow reaches a regime which is nearly steady-state, if the initial perturbation is a pure sinusoid. The bubbles rise at constant velocity. If $A \simeq 1$, we can carry out an approximate analysis of the resulting flow pattern (following Davies and Taylor 1950, incorporating a suggestion by Layzer 1955) and determine the velocity of the tip of the bubble. Layzer considers the entire history of the instability, from the initial linear stage to the asymptotic steady state, but we focus only on the latter here.

To do so, we employ the concept of potential flow. The law of conservation of circulation implies that for isentropic flows (that is, flows which are not dissipating or exchanging energy or subjected to shock waves), the curl of the velocity field, $\nabla \times \mathbf{v}$ (called the vortic$i t y$ ) is constant along particle trajectories. In particular, if the vorticity vanishes anywhere on a fluid trajectory, it vanishes everywhere on the trajectory. In the case of an array of bubbles rising into initially motionless fluid, the vorticity of the fluid at a large distance above the bubbles is zero because the fluid is at rest. Even after the fluid begins to fall past the bubbles, its vorticity remains zero, by the law of conservation of circulation. Like any 
vector field whose curl is zero, the velocity can be therefore be expressed as the gradient of some scalar, by virtue of the vector identity $\nabla \times(\nabla \phi)=0$. This scalar is called the velocity potential, and we write $\mathbf{v}=\nabla \phi$. This kind of flow is termed potential flow, or irrotational flow.

If furthermore we assume that the flow is incompressible, as we did in the linear analysis of Rayleigh-Taylor instability, we have that the velocity is divergenceless: $\nabla \cdot \mathbf{v}=0$. (This follows from the vanishing of the Lagrangian total derivative, Eq. (A-11), and the continuity equation, Eq. (2).) Therefore, expressing the velocity as the gradient of the potential, we conclude that, for incompressible potential flow, the velocity potential satisfies Laplace's equation:

$$
\nabla^{2} \phi=0 .
$$

Determining the flow field for an array of rising bubbles then amounts to solving Laplace's equation subject to the appropriate boundary conditions.

Another useful relationship for problems of this type is given by Bernoulli's equation. It states that, for steady flow of an incompressible fluid,

$$
\frac{1}{2} v^{2}+\frac{p}{\rho}+g z=\text { constant }
$$

along particle trajectories. In our problem, in which $A \simeq 1$, it is a reasonable approximation to take $p=$ constant within the low-density bubble near its tip. Since the high-density fluid at the bubble surface must be in pressure equilibrium with the bubble, and since density is constant in incompressible flow, we can assume that along the surface of the bubble

$$
\frac{1}{2} v^{2}+g z=\text { constant. }
$$

Let us consider an exactly sinusoidal initial perturbation at an interface, with arbitrary values of the wavevector components $k_{x}$ and $k_{y}$. By appropriately rotating the coordinate system in the $(\hat{\mathbf{x}}, \hat{\mathbf{y}})$ plane we can make the $\hat{\mathbf{x}}$ direction coincide with the direction of the wavevector $\mathbf{k}$, so that $k_{y}=0$ and $k_{x}=k$. Thus the sinusoid varies only in $\hat{\mathbf{x}}$, and we can ignore the $\hat{\mathbf{y}}$ direction in the following analysis.

From this sort of initial condition, a flow field will eventually arise consisting of an array of identical rising bubbles (which are two-dimensional, like long tunnels, having no variation in $\hat{\mathbf{y}}$ ) arranged with a spatial period of $\lambda=2 \pi / k$. The flow pattern is the same as that for a single bubble rising between two parallel frictionless walls located at $x= \pm \lambda / 2$. The boundary condition at the walls is that the component of the flow velocity normal to the walls vanish there:

$$
v_{x}\left(x= \pm \frac{\lambda}{2}\right)=0 .
$$

We now transform to the frame-of-reference rising at the same speed as the bubble. Call this speed $U$; the point of this analysis is to determine the value of $U$. An additional boundary condition is that in the frame of the bubble, the undisturbed fluid far above the bubble is traveling downward at velocity

$$
v_{z}(z=+\infty)=-U .
$$


Solutions of Laplace's equation are well-known from many branches of physics. For geometries such as in our bubble problem, where the flow is two-dimensional and confined by planar walls, it is clear that a potential of the form

$$
\phi(x, z)=-z U-\sum_{n=1}^{\infty} \frac{\lambda a_{n}}{2 n \pi} e^{-\frac{2 n \pi z}{\lambda}} \cos \left(\frac{2 n \pi x}{\lambda}\right)
$$

satisfies Laplace's equation and the boundary conditions just defined. For

$$
\begin{gathered}
\frac{\partial \phi}{\partial x}=\sum_{n=1}^{\infty} a_{n} e^{-\frac{2 n \pi z}{\lambda}} \sin \left(\frac{2 n \pi x}{\lambda}\right) \\
\frac{\partial \phi}{\partial z}=-U+\sum_{n=1}^{\infty} a_{n} e^{-\frac{2 n \pi z}{\lambda}} \cos \left(\frac{2 n \pi x}{\lambda}\right) \\
\frac{\partial^{2} \phi}{\partial x^{2}}=\sum_{n=1}^{\infty} \frac{2 n \pi}{\lambda} a_{n} e^{-\frac{2 n \pi z}{\lambda}} \cos \left(\frac{2 n \pi x}{\lambda}\right) \\
\frac{\partial^{2} \phi}{\partial z^{2}}=-\sum_{n=1}^{\infty} \frac{2 n \pi}{\lambda} a_{n} e^{-\frac{2 n \pi z}{\lambda}} \cos \left(\frac{2 n \pi x}{\lambda}\right)
\end{gathered}
$$

Thus

$$
\frac{\partial^{2} \phi}{\partial x^{2}}+\frac{\partial^{2} \phi}{\partial z^{2}}=0
$$

as Laplace's equation requires. (Recall that for our choice of coordinate axes, $\partial \phi / \partial y=$ $\partial^{2} \phi / \partial y^{2} \equiv 0$.) Furthermore $v_{x}=\partial \phi / \partial x=0$ at $x= \pm \frac{\lambda}{2}$ and $v_{z}=\partial \phi / \partial z \rightarrow-U$ as $z \rightarrow \infty$, as our boundary conditions require.

The trajectory of any fluid particle in the flow field is described by the stream function $\psi$, which is related to the velocity potential by

$$
\begin{gathered}
\frac{\partial \psi}{\partial x}=\frac{\partial \phi}{\partial z} \\
\frac{\partial \psi}{\partial z}=\frac{-\partial \phi}{\partial x} .
\end{gathered}
$$

It is easy to verify that the function

$$
\psi(x, z)=-x U+\sum_{n=1}^{\infty} \frac{\lambda a_{n}}{2 n \pi} e^{-\frac{2 n \pi z}{\lambda}} \sin \left(\frac{2 n \pi x}{\lambda}\right)
$$

satisfies these relationships. The stream function is constant along particle trajectories for steady flow, so that trajectories are given implicitly by

$$
\psi(x, z)=\psi_{c}=\text { constant }
$$


At $z=+\infty$, the stream function becomes $\psi(x, \infty)=-x U$, so we see that $\psi_{c}$ is related to the distance of the trajectory from the symmetry plane $x=0$ at large distance above the bubble. Thus the trajectory for a fluid particle that flows down the plane $x=0$ and then along the boundary of the bubble is given by $\psi_{c}=0$, which implies that

$$
\frac{1}{x U} \sum_{n=1}^{\infty} \frac{\lambda a_{n}}{2 n \pi} e^{-\frac{2 n \pi z}{\lambda}} \sin \left(\frac{2 n \pi x}{\lambda}\right)=1
$$

is the equation of the bubble surface.

A simple approximation to the solution of this problem is obtained, following Davies and Taylor (1950), by keeping only the first term in the sum defining $\phi$ and $\psi$. That is, using $k=2 \pi / \lambda$,

$$
\begin{aligned}
& \phi=-z U-\frac{a_{1}}{k} e^{-k z} \cos k x, \\
& \psi=-x U+\frac{a_{1}}{k} e^{-k z} \sin k x .
\end{aligned}
$$

Then the velocity components are

$$
\begin{gathered}
v_{x}=\frac{\partial \phi}{\partial x}=-\frac{\partial \psi}{\partial z}=a_{1} e^{-k z} \sin k x \\
v_{z}=\frac{\partial \phi}{\partial z}=\frac{\partial \psi}{\partial x}=-U+a_{1} e^{-k z} \cos k x .
\end{gathered}
$$

The surface of the bubble is given by

$$
e^{-k z}=\frac{U k x}{a_{1} \sin k x}
$$

The height of the apex of the bubble, at $x=0$, is determined by the values of $a_{1}$ and $U$. If we demand that the apex occur at $z=0$, then we must have $a_{1}=U$ and so the bubble surface is given by

or

$$
e^{-k z}=\frac{k x}{\sin k x}
$$

$$
z=\frac{1}{k} \ln \left(\frac{\sin k x}{k x}\right) .
$$

To proceed with the solution, we now require that Bernoulli's equation be satisfied along the bubble surface. Inserting the above expressions for the velocity components into Eq. (C-1), with $a_{1}=U$, leads to

$$
U^{2}\left(e^{-2 k z} \sin ^{2} k x+1-2 e^{-k z} \cos k x+e^{-2 k z} \cos ^{2} k x\right)+2 g z=\text { constant, }
$$

or

$$
e^{-2 k z}+1-2 e^{-k z} \cos k x+\frac{2 g z}{U^{2}}=0
$$


We choose the constant to be zero on the right-hand side of the equation because the apex of the bubble is a stagnation point, with $v_{x}=v_{y}=0$, and its height is $z=0$. Thus it is clear from Eq. (C-1) that the constant is zero for this trajectory. Along the bubble surface, Eq. (C-2) applies, so we insert that condition into Eq. (C-3). The result is

$$
\frac{(k x)^{2}}{\sin ^{2} k x}-2 \frac{k x}{\tan k x}+1+\frac{2 g}{k U^{2}} \ln \left(\frac{\sin k x}{k x}\right)=0
$$

or

$$
u^{2} \tan u-2 u \sin ^{2} u+\sin ^{2} u \tan u+\frac{2 g}{k U^{2}} \sin ^{2} u \tan u \ln \left(\frac{\sin u}{u}\right)=0,
$$

where $u \equiv k x$.

Now, for any particular choice of $g, k$, and $U$, this expression can only be satisfied exactly at a single value of $x$ in addition to $x=0$. It cannot be satisfied over the entire bubble surface. This is a consequence of having chosen the simplified potential and stream functions with only the first term of the sum. Nevertheless we can determine a reasonably accurate value for $U$ by requiring that Eq. (C-4) be satisfied in a first-order neighborhood of $x=0$. Accordingly we expand the functions in Eq. (C-4):

$$
\begin{gathered}
\sin ^{2} u=u^{2}-\frac{u^{4}}{3}+O\left[u^{6}\right] ; \\
\tan u=u+\frac{u^{3}}{3}+O\left[u^{5}\right] ; \\
\sin ^{2} u \tan u=u^{3}+O\left[u^{7}\right] ; \\
\ln \left(\frac{\sin u}{u}\right)=-\frac{u^{2}}{6}-\frac{u^{4}}{180}+O\left[u^{6}\right] ; \\
\sin ^{2} u \tan u \ln \left(\frac{\sin u}{u}\right)=-\frac{u^{5}}{6}+O\left[u^{7}\right] .
\end{gathered}
$$

So Eq. (C-4) becomes

$$
u^{3}+\frac{u^{5}}{3}-2 u^{3}+\frac{2 u^{5}}{3}+u^{3}-\frac{2 g}{k U^{2}} \frac{u^{5}}{6}+O\left[u^{7}\right]=0
$$

implying that

or

$$
u^{5}\left(\frac{1}{3}+\frac{2}{3}-\frac{g}{3 k U^{2}}\right)=0
$$

$$
U=\sqrt{\frac{g}{3 k}}=\sqrt{\frac{g \lambda}{6 \pi}} \simeq 0.2303 \sqrt{g \lambda}
$$

This is exactly the result of Layzer (1955) for the case of asymptotic steady-state twodimensional flow between parallel walls. He takes as the length scale the half-distance between the walls $a=\lambda / 2$, so that he writes

$$
U=\sqrt{\frac{g a}{3 \pi}} \simeq 0.3257 \sqrt{g a} .
$$


Layzer also considers the flow of a bubble of circular cross-section, contained in a tube of radius $R$. He obtains in this case

$$
U=\frac{1}{\sqrt{\beta_{1}}} \sqrt{g R} \simeq 0.5108 \sqrt{g R},
$$

where $\beta_{1} \simeq 3.832$ is the first zero of the Bessel function $J_{1}$.

Thus we see that larger bubbles rise faster than smaller bubbles. This dependence is opposite to that for the linear stage of the instability, in which we found that smaller wavelengths grow faster than larger wavelengths.

This analysis assumes Atwood number $A \simeq 1$. For smaller values of $A$, Ofer et al. (1992) have found numerically that

$$
U=\alpha \sqrt{\frac{2 A}{1+A} g \lambda},
$$

where $0.2 \leq \alpha \leq 0.3$.

Shvarts (1994) points out that this expression is a consequence of the balance between buoyancy and drag acting on the rising bubble. Consider a spherical bubble of radius $R$ and density $\rho_{L}$ rising at constant velocity in an initially motionless medium of higher density $\rho_{H}$. The buoyancy force is $F_{b}=\left(\rho_{H}-\rho_{L}\right) V g$, where $g$ is the acceleration of gravity and $V$ is the volume of the bubble. If the bubble is rising at a velocity $U$, then the drag force is $F_{d}=C_{d} S \rho_{H} U^{2}$, where $C_{d}$ is a drag coefficient and $S=\pi R^{2}$ is the cross-sectional area of the bubble. The drag force depends only on the ambient density $\rho_{H}$, not the bubble density $\rho_{L}$, because the drag force results from the increase of momentum of the ambient fluid, which is initially motionless; the bubble's momentum does not change, owing to the balance of forces acting on it. Equating the forces gives

$$
\left(\rho_{H}-\rho_{L}\right) V g=C_{d} S \rho_{H} U^{2}
$$

or

$$
U^{2}=\frac{1}{C_{d}} \frac{V}{S} \frac{\left(\rho_{H}-\rho_{L}\right)}{\rho_{H}} g .
$$

Define the Atwood number $A$ as

$$
A \equiv \frac{\left(\rho_{H}-\rho_{L}\right)}{\left(\rho_{H}+\rho_{L}\right)} .
$$

Then

$$
\frac{\left(\rho_{H}-\rho_{L}\right)}{\rho_{H}}=\frac{2 A}{1+A} .
$$

Also $V / S=4 R / 3$. If we define a wavelength $\lambda=2 R$, to make the correspondence with the Rayleigh-Taylor bubble considered above, then $V / S=2 \lambda / 3$. So finally

$$
U=\sqrt{\frac{2}{3 C_{d}}} \sqrt{\frac{2 A}{1+A} g \lambda},
$$


analogous to the form shown above.

\section{Saturation and multimode interactions in intermediate-stage Rayleigh- Taylor instability}

\section{Saturation threshold}

The linear analysis of Sec. A depends on the validity of the small-amplitude assumption, that is, on the extent to which first-order quantities are in fact much smaller than the corresponding zeroth-order quantities. However, if the exponential growth that characterizes the linear stage were to persist long enough, the small-amplitude assumption would eventually be violated for any initial perturbation, however small. The departure of the instability evolution from linearity is called saturation. We can estimate the conditions required for linearity by considering, for example, the first-order acceleration of a sinusoidal perturbation mode and its relation to the zeroth-order acceleration of gravity $g$. The $z$-velocity of a pure mode with wavevector $\mathbf{k}$ oriented along $\hat{\mathbf{x}}$ can be described by

$$
v_{z}(x, z, t)=W(z) e^{\gamma t} \cos k x,
$$

so that the displacement of the interface (initially at $z=0$ ) is

$$
\eta(x, t)=\int_{0}^{t} v_{z}\left(x, 0, t^{\prime}\right) d t^{\prime}=\frac{1}{\gamma} v_{z}(x, 0, t) .
$$

The acceleration of the interface is

$$
\frac{\partial v_{z}(x, 0, t)}{\partial t}=\gamma v_{z}(x, 0, t)=\gamma^{2} \eta(x, t) .
$$

Linearity requires that this acceleration be much smaller than gravity: $\gamma^{2} \eta<<g$. Since the linear growth rate $\gamma=\sqrt{k g A}$, this is $A k \eta<<1$. Since $A \leq 1$, a sufficient condition for linearity is simply

$$
k \eta<<1 .
$$

In terms of the wavelength of the mode, this condition is

$$
\eta<<\lambda / 2 \pi \simeq 0.16 \lambda .
$$

The consequence of saturation is that the growth of the instability is no longer exponential, but begins to approach the constant-velocity bubble rise typical of late-stage growth. A more stringent estimate of the requirement for linearity comes from estimating the interface displacement at which the linear-stage interface velocity equals the late-stage bubble velocity. As we have just seen, the interface velocity is $v_{z}(x, 0, t)=\gamma \eta$, while the bubble velocity is $\sqrt{g / 3 k}$. Equating these, for $A=1$, gives $k \eta=1 / \sqrt{3} \simeq 0.58$, so that linearity requires

$$
\eta<\frac{1}{\sqrt{3}} \frac{\lambda}{2 \pi} \simeq 0.09 \lambda \text {. }
$$


Another consequence of the onset of nonlinearity is that separate perturbation modes on the interface, which grow as if they are isolated during the linear stage, begin to notice one another's presence. This occurs because they begin to affect the zeroth-order flow field which drives the instability; for example, a short-wavelength mode riding along on the bubble of a long-wavelength mode experiences a different effective gravity than the initial $\mathbf{g}$, because of the additional acceleration in the long-wavelength bubble. This interaction is called mode coupling.

\section{Haan saturation model}

Real surfaces in actual ICF experiments have structure at many scales, from millimeters to angstroms. The structure arises for a variety of reasons, including the inherent heterogeneous crystalline structure of materials, as well as marks left by fabrication and machining. When Fourier analyzed, the surfaces typically have a full spectrum, with spectral power at all modes up to some very high wavenumber. An important question arises concerning how saturation occurs in a full spectrum, as opposed to the case of a pure mode just discussed. This is because a group of modes with nearly equal wave vectors can combine constructively over a region of the surface, producing a structure whose net amplitude is much larger than the modes' individual amplitudes. It seems clear that the saturation of this structure should occur when its net amplitude is about $10 \%$ of its effective wavelength, as discussed above for pure sinusoids. This means that the individual modes summing to produce this structure must saturate a good deal earlier than we would expect if they were isolated from other modes and individually obeying the inequality (D-1). A prescription for determining when modes saturate in a full spectrum was developed by Haan (1989) and is known as the Haan saturation model. It expresses a type of modal interaction which is a short-range interaction in wavevector space, involving neighboring modes which stay in phase over a large enough region to form a structure of significantly higher amplitude than any of the individual modes.

The basic conceptual point of the Haan model is that a pure mode cannot be distinguished from a superposition of several modes except by measurements over a sufficiently large spatial region; the region must be large enough that the individual modes in the superposition have gone out of phase. In regions smaller than this, the saturation of the multimode superposition must occur in the same way as the saturation of the pure mode. For example, consider two modes of nearly equal wavelength [say $\lambda$ and $\lambda(1+\delta)$ ], equal amplitudes, and parallel wavevectors. The modes stay in phase for a large distance because their wavelengths are so nearly equal. Where they are in phase, they combine to create a net perturbation whose amplitude is twice the individual amplitudes and whose wavelength is approximately $\lambda$. When the net perturbation saturates, the two superposed modes clearly have amplitudes which are about half the value of the single-mode saturation amplitude, yet they must individually saturate. In this sense, they are interacting because they are affecting each other's saturation; they are reducing the saturation threshold by a factor of two.

The Haan model generalizes this idea to the case of many modes with various wavevectors. Two parameters play a role in the model. One of these, $\zeta$, denotes the amplitude threshold for the saturation of a single isolated mode, in the sense that a mode is linear only when its amplitude $\eta<<\zeta \lambda$. We saw above that $\zeta$ is in the range $\sim 0.09$ to $\sim 0.16$. 
The other parameter, $\epsilon$, describes the range in wavenumber space over which modes stay in phase long enough to interact by affecting one another's saturation. The model assumes that, if we consider a band of modes centered around wavevector $\mathbf{k}$, then the modes $\mathbf{k}^{\prime}$ which interact have $\left|\mathbf{k}-\mathbf{k}^{\prime}\right|<\epsilon k$. That is, the interacting modes occupy a circle of radius $\epsilon$ in wavenumber space, centered on wavevector $\mathbf{k}$. These modes combine to create a physical structure with a root-mean-square ( $\mathrm{rms}$ ) amplitude that we shall call $\sigma$, and a wavelength approximately equal to $\lambda=2 \pi / k$. We expect the structure to saturate when $\sqrt{2} \sigma \simeq \zeta \lambda$, where the factor $\sqrt{2}$ converts the rms amplitude to the peak amplitude. By evaluating $\sigma$ in terms of the individual modes' amplitudes $S(k)$, we can determine $S(k)$ at saturation in terms of the parameters $\zeta$ and $\epsilon$.

The rms amplitude $\sigma$ of a band of Fourier modes is the root of the sum of the squared amplitudes of the individual modes. Thus the interacting modes have

$$
\begin{aligned}
& \sigma^{2}=\sum_{\left|\mathbf{k}^{\prime}-\mathbf{k}\right|<\epsilon k} S^{2}\left(k^{\prime}\right) \\
& \simeq N(k, \epsilon) S^{2}(k) \\
& \simeq A(k, \epsilon) D(k) S^{2}(k)
\end{aligned}
$$

where $N(k, \epsilon)$ is the number of modes in the circle in $k$-space, $A(k, \epsilon)$ is the area of the circle, and $D(k)$ is the density of states.

If we consider a planar interface whose extent is $0 \leq x \leq L$ and $0 \leq y \leq L$, then the components of $\mathbf{k}^{\prime}$ have discrete allowed values $k_{x}^{\prime}=2 \pi n / L, k_{y}^{\prime}=2 \pi m / L$, for $m, n=$ $1, \ldots, \infty$. Thus there is a mode spaced every $2 \pi / L$ wavenumber units along both axes in wavenumber space, so the density of states is $D(k)=L^{2} /(2 \pi)^{2}$. The area of the circle is $2 \pi(\epsilon k)^{2}$, so that

$$
N(k, \epsilon)=(\epsilon k L)^{2} / 2 \pi
$$

Therefore

$$
\sigma=\left[(1 / 2 \pi)(\epsilon k L)^{2} S^{2}(k)\right]^{1 / 2}
$$

The saturation condition

$$
\zeta \lambda=\sqrt{2} \sigma=\left[(1 / \pi)(\epsilon k L)^{2} S^{2}(k)\right]^{1 / 2}
$$

can then be solved for $S(k)$, the modal amplitude at saturation. The result is

$$
S(k)=2 \pi^{3 / 2}(\zeta / \epsilon)\left(1 / L k^{2}\right)
$$

which can also be written

$$
S(k)=\frac{1}{2 \sqrt{\pi} \epsilon} \frac{\zeta \lambda}{(L / \lambda)}
$$

Compared to the saturation amplitude for isolated pure modes $S_{i s o}(k)=\zeta \lambda$, this result predicts that saturation occurs at much lower amplitudes for a full spectrum, especially for high modes, where $L / \lambda>>1$. 
Haan (1989) defines a parameter

$$
\nu \equiv 2 \pi^{3 / 2}(\zeta / \epsilon)
$$

which combines the parameters of interest into a single number. Thus the saturation amplitude is

$$
S(k)=\nu /\left(L k^{2}\right)=\left(\nu / 4 \pi^{2}\right)(\lambda / L) \lambda .
$$

From fitting predictions of the model to experiments, the value of $\nu$ is determined to be $\sim 4$.

To generalize this result to spherical geometry, we adopt the following equivalence for spherical-harmonic mode number $l$ and spherical radius $R$ :

$$
k^{2}=l(l+1) / R^{2} \simeq l^{2} / R^{2}
$$

Then the saturation condition becomes

$$
\frac{\nu}{L k^{2}} \simeq \frac{\nu}{R\left(l^{2} / R^{2}\right)}=\frac{\nu R}{l^{2}} .
$$

The other element of the Haan saturation model is a prescription for the behavior of modes after they cross the saturation threshold, that is, after their amplitudes reach the value $S(k)=\nu /\left(L k^{2}\right)$. Let $\eta_{l i n}(k, t)$ be the time-dependent amplitude a mode would have if saturation were not an issue, that is, if the mode remained linear. Then the amplitude after saturation is taken to be

$$
\eta_{s a t}(k, t)=S(k)\left[1+\ln \frac{\eta_{l i n}(k, t)}{S(k)}\right] .
$$

In the case of a planar density discontinuity with a constant acceleration $g$ and Atwood number $A$ (that is, the classical incompressible Rayleigh-Taylor problem), we saw earlier that $\eta_{l i n}(k, t)=\eta_{0} e^{\gamma t}$, where $\gamma=\sqrt{k g A}$ and $\eta_{0}$ is the initial perturbation amplitude of an isolated single mode with wavenumber $k$ on the interface. For this case,

$$
\eta_{s a t}(k, t)=S(k)\left[1+\ln \eta_{0}+\gamma t-\ln S(k)\right] .
$$

The growth velocity of the saturated mode is constant in time,

$$
d \eta_{s a t} / d t=S(k) \gamma,
$$

and is equal to the growth velocity of the linear perturbation at the time of saturation. In general, for non-constant accelerations,

$$
\frac{d \eta_{\text {sat }}}{d t}=\frac{S(k)}{\eta_{\text {lin }}} \frac{d \eta_{\text {lin }}}{d t} .
$$




\section{E. Instability calculations in ICF implosions}

Several methods have been used in recent years to calculate the growth of hydrodynamic instabilities in ICF implosions. To represent reality as accurately as possible, the methods must account for time-varying accelerations, continuous gradients in density and velocity, and energy transport. Historically, linearized perturbation codes were used first, but recently nonlinear radiation-hydrodynamic simulation codes have been used quite widely and routinely.

\section{Linearized perturbation codes}

The linearized equations of mass and momentum conservation were derived in an earlier section. For realistic flows, we must also consider an energy equation. For example (Scannapieco and Cranfill 1978), we begin with the first law of thermodynamics, which specifies that a change $d E$ in the specific internal energy $E$ of a fluid element may arise from two sources. There is a contribution $-p d V$ when the specific volume $V=1 / \rho$ of the fluid element changes by an amount $-d V$ at a pressure $p$, (a volume decrease corresponding to an energy increase). Additionally there may be a contribution $T d S$ when the specific entropy $S$ of the element changes by an amount $d S$ at temperature $T$. If the change occurs in an interval of time $d t$, then

$$
\frac{d E}{d t}=T \frac{d S}{d t}+\frac{p}{\rho^{2}} \frac{d \rho}{d t}
$$

where $d \rho / \rho^{2}=-d V$. The entropy change is given by the equation of heat transfer

$$
T \frac{d S}{d t}=\frac{1}{\rho} \nabla \cdot[\kappa(\rho, T) \nabla T]+\frac{\dot{q}(\rho, T)}{\rho} .
$$

The first term on the right-hand side represents heating arising from the divergence of a flux of thermal radiation and conduction; the combined thermal and grey-radiative conductivity is denoted by $\kappa(\rho, T)$. The second term on the right-hand side represents heating by an energy source, which could be thermonuclear reactions or a laser. The heating rate is $\dot{q}(\rho, T)$. Here $T$, the material temperature, is assumed equal for ions and electrons.

Inserting this equation in the preceding equation leads to

$$
\frac{d E}{d t}=\frac{p}{\rho^{2}} \frac{d \rho}{d t}+\frac{1}{\rho} \nabla \cdot[\kappa(\rho, T) \nabla T]+\frac{\dot{q}(\rho, T)}{\rho} .
$$

With an equation-of-state, we can eliminate one of the thermodynamic variables in terms of two of the others, for example

$$
E=E(\rho, T)
$$

Then the energy equation becomes

$$
\left(\frac{\partial E}{\partial T}\right)_{\rho} \frac{d T}{d t}+\left[\left(\frac{\partial E}{\partial \rho}\right)_{T}-\frac{p}{\rho^{2}}\right] \frac{d \rho}{d t}=\frac{1}{\rho} \nabla \cdot[\kappa(\rho, T) \nabla T]+\frac{\dot{q}(\rho, T)}{\rho} .
$$

In the manner of Scannapieco and Cranfill (1978), we collect the basic zeroth-order hydrodynamic equations here, using Eqs. (1) and (2) for our continuity (that is, mass) 
and momentum equations, respectively, omitting gravity in the ICF context. The energy equation has been expressed as a temperature equation.

$$
\begin{gathered}
\frac{\partial \rho}{\partial t}+\nabla \cdot(\rho \mathbf{v})=0, \\
\rho \frac{\partial \mathbf{v}}{\partial t}+\rho(\mathbf{v} \cdot \nabla) \mathbf{v}=-\nabla \mathrm{p}, \\
\left(\frac{\partial E}{\partial T}\right)_{\rho} \frac{d T}{d t}=\left[\frac{p}{\rho^{2}}-\left(\frac{\partial E}{\partial \rho}\right)_{T}\right] \frac{d \rho}{d t}+\frac{1}{\rho} \nabla \cdot[\kappa \nabla T]+\frac{\dot{q}}{\rho}, \\
p=p(\rho, T), \\
E=E(\rho, T), \\
\kappa=\kappa(\rho, T), \\
\dot{q}=\dot{q}(\rho, T) .
\end{gathered}
$$

The first-order linearized equations are obtained from the hydrodynamic equations Eqs. (E-1) through (E-7) in the same manner as in the discussion of Rayleigh-Taylor instability. Physical variables are represented as a sum of a zeroth-order part, which satisfies the hydrodynamic equations, and a small first-order part. Thus

$$
\begin{array}{r}
\rho=\rho_{0}+\rho_{1}, \\
\mathbf{v}=\mathbf{v}_{0}+\mathbf{v}_{1}, \\
p=p_{0}+p_{1}, \\
T=T_{0}+T_{1},
\end{array}
$$

where the equation of state allows us to write

$$
p_{1}=\left(\frac{\partial p}{\partial T}\right)_{\rho 0} T_{1}+\left(\frac{\partial p}{\partial \rho}\right)_{T 0} \rho_{1} .
$$

Substitution of these expressions into Eqs. (E-1) through (E-7) yields, after subtracting the zeroth-order equations and linearizing [following Scannapieco and Cranfill (1978), who extended a treatment by McCrory et al. (1977)],

$$
\begin{gathered}
\frac{\partial \rho_{1}}{\partial t}+\nabla \cdot\left(\rho_{1} \mathbf{v}_{0}+\rho_{0} \mathbf{v}_{1}\right)=0 \\
\rho_{0}\left(\frac{\partial \mathbf{v}_{1}}{\partial t}+\mathbf{v}_{1} \cdot \nabla \mathbf{v}_{0}+\mathbf{v}_{0} \cdot \nabla \mathbf{v}_{1}\right)=\frac{\rho_{1}}{\rho_{0}} \nabla p_{0}-\nabla p_{1}, \\
\left(\frac{\partial E}{\partial T}\right)_{\rho 0}\left(\frac{d T_{1}}{d t}+\mathbf{v}_{1} \cdot \nabla T_{0}\right)+\left(\frac{\partial E}{\partial T}\right)_{\rho 1} \frac{d T_{0}}{d t}=
\end{gathered}
$$




$$
\begin{gathered}
{\left[\frac{p_{0}}{\rho_{0}^{2}}-\left(\frac{\partial E}{\partial \rho}\right)_{T 0}\right]\left(\frac{d \rho_{1}}{d t}+\mathbf{v}_{1} \cdot \nabla \rho_{0}\right)+\left[\frac{p_{1}}{\rho_{0}^{2}}-\frac{2 p_{0}}{\rho_{0}^{3}} \rho_{1}-\left(\frac{\partial E}{\partial \rho}\right)_{T 1}\right] \frac{d \rho_{0}}{d t}} \\
+\frac{1}{\rho_{0}} \nabla \cdot\left(\kappa_{0} \nabla T_{1}+\kappa_{1} \nabla T_{0}\right)+\frac{\dot{q}_{1}}{\rho_{0}}-\frac{\rho_{1}}{\rho_{0}^{2}}\left(\dot{q}_{0}+\nabla \cdot \kappa_{0} \nabla T_{0}\right),
\end{gathered}
$$

where

$$
\begin{gathered}
p_{1}=\left(\frac{\partial p}{\partial T}\right)_{\rho 0} T_{1}+\left(\frac{\partial p}{\partial \rho}\right)_{T 0} \rho_{1}, \\
\kappa_{1}=\left(\frac{\partial \kappa}{\partial T}\right)_{\rho 0} T_{1}+\left(\frac{\partial \kappa}{\partial \rho}\right)_{T 0} \rho_{1}, \\
\dot{q}_{1}=\left(\frac{\partial \dot{q}}{\partial T}\right)_{\rho 0} T_{1}+\left(\frac{\partial \dot{q}}{\partial \rho}\right)_{T 0} \rho_{1}, \\
\left(\frac{\partial E}{\partial T}\right)_{\rho_{1}}=\left[\frac{\partial}{\partial T}\left(\frac{\partial E}{\partial T}\right)\right]_{\rho 0} T_{1}+\left[\frac{\partial}{\partial \rho}\left(\frac{\partial E}{\partial T}\right)\right]_{\rho 0} \rho_{1} \\
\left(\frac{\partial E}{\partial \rho}\right)_{T 1}=\left[\frac{\partial}{\partial T}\left(\frac{\partial E}{\partial \rho}\right)_{T}\right]_{\rho 0} T_{1}+\left[\frac{\partial}{\partial \rho}\left(\frac{\partial E}{\partial \rho}\right)_{T}\right]_{T 0} \rho_{1} .
\end{gathered}
$$

Equation (E-8) is the same as Eq. (A-7), and Eq. (E-9) is the same as Eq. $\left({ }^{*} \mathrm{c}\right)$, that is, they are the same as Eqs. (A-9) and (A-10) when $\mathbf{v}_{0}$ does not vanish and gravity is negligible.

Scannapieco and Cranfill (1978) recast these equations in Lagrangian form, so that the first-order quantities are associated with mass elements in the flow rather than fixed points in space. This is convenient when the zeroth-order quantities are computed in a Lagrangian code, and it is desired to evaluate the first-order flow at the corresponding Lagrangian mass elements.

To do this, consider that the first-order perturbation in the flow causes a displacement in the position of a fluid element from its zeroth-order position $\mathbf{r}_{0}$ to a new position $\mathbf{R}$. The magnitude of displacement is the displacement vector

$$
\mathbf{X} \equiv \mathbf{R}-\mathbf{r}_{0} .
$$

The relationship between the Lagrangian and Eulerian first-order terms is found by considering some physical variable $\phi$. In an Eulerian reference frame, we write as we have done before

$$
\phi\left(\mathbf{r}_{0}, t\right)=\phi_{0}\left(\mathbf{r}_{0}, t\right)+\phi_{1}^{E}\left(\mathbf{r}_{0}, t\right),
$$

where $\phi_{1}^{E}$ is the Eulerian perturbation. In a Lagrangian frame, we need to know the value of variables at the new position $\mathbf{R}$ :

$$
\phi(\mathbf{R}, t)=\phi_{0}\left(\mathbf{r}_{0}, t\right)+\phi_{1}^{L}(\mathbf{R}, t),
$$

where $\phi_{1}^{L}$ is the Lagrangian perturbation. Eliminating $\phi_{0}$ between these equations, we have

$$
\phi\left(\mathbf{r}_{0}, t\right)-\phi_{1}^{E}\left(\mathbf{r}_{0}, t\right)=\phi(\mathbf{R}, t)-\phi_{1}^{L}(\mathbf{R}, t) .
$$


The change in $\phi$ between $\mathbf{r}_{0}$ and $\mathbf{R}$ is given, for small displacements, by the Taylor-series expansion for $\phi$ at the position $\mathbf{r}_{0}$ :

$$
\phi(\mathbf{R}, t) \simeq \phi\left(\mathbf{r}_{0}, t\right)+\mathrm{X} \cdot \nabla \phi\left(\mathbf{r}_{0}, t\right) \simeq \phi\left(\mathbf{r}_{0}, t\right)+\mathbf{X} \cdot \nabla \phi_{0}\left(\mathbf{r}_{0}, t\right)
$$

where we have neglected terms proportional to $\mathrm{X} \cdot \nabla \phi_{1}$ and $\mathrm{X}^{2}$ and higher order. (Here $\phi_{1}$ represents either $\phi_{1}^{E}$ or $\phi_{1}^{L}$.) So we see that

$$
\phi(\mathbf{R}, t)-\phi\left(\mathbf{r}_{0}, t\right) \simeq \mathbf{X} \cdot \nabla \phi_{0}\left(\mathbf{r}_{0}, t\right) .
$$

From Eq. (E-11)

$$
\phi_{1}^{L}(\mathbf{R}, t)-\phi_{1}^{E}\left(\mathbf{r}_{0}, t\right)=\phi(\mathbf{R}, t)-\phi\left(\mathbf{r}_{0}, t\right)
$$

so that finally

$$
\phi_{1}^{L}(\mathbf{R}, t) \simeq \phi_{1}^{E}\left(\mathbf{r}_{0}, t\right)+\mathbf{X} \cdot \nabla \phi_{0}\left(\mathbf{r}_{0}, t\right) .
$$

The Eulerian first-order equations may be converted to Lagrangian first-order equations by the use of Eq. (E-12). An additional equation for the new variable $\mathbf{X}$ is required to close the system:

$$
\frac{d \mathbf{X}}{d t}=\mathbf{v}_{1}^{L}
$$

where $\mathbf{v}_{1}^{L}$ is the Lagrangian first-order velocity.

At this point, Scannapieco and Cranfill (1978) assume that the zeroth-order flow is spherically symmetric, and express the Lagrangian first-order equations in component form. Then they perform a spherical-harmonic decomposition of the component equations, arriving at the following set of equations for the spherical-harmonic amplitudes. Each of the variables with superscript $l$ in the following equations represents the spherical-harmonic amplitude in mode $l$ for the corresponding perturbation variable.

$$
\begin{gathered}
\rho_{1}^{l}=\rho_{1 I}^{l}-\rho_{0}\left[\frac{2 B^{l}}{r}+\frac{\partial B^{l}}{\partial r}+D^{l}\right] \\
\frac{d B^{l}}{d t}=A^{l} \\
\frac{d D^{l}}{d t}=\frac{C^{l}}{r^{2}} \\
\frac{d A^{l}}{d t}=-\frac{1}{\rho_{0}}\left[\frac{\partial p_{1}^{l}}{\partial r}+\left(\frac{2 B^{l}}{r}+D^{l}\right) \frac{\partial p_{0}}{\partial r}\right]+\frac{\rho_{1 I}^{l} \cdot \frac{\partial p_{0}}{\rho_{0}^{2}}}{\partial r} \\
\frac{d C^{l}}{d t}=\frac{l(l+1)}{\rho_{0}}\left[p_{1}^{l}-B^{l} \frac{\partial p_{0}}{\partial r}\right] \\
\left(\frac{\partial E}{\partial T}\right)_{\rho 0} \frac{d T_{1}^{l}}{d t}+\left(\frac{\partial E}{\partial T}\right)_{\rho 1}^{l} \frac{d T_{0}}{d t}=-\frac{\rho_{1}^{l}}{\rho_{0}^{2}} \frac{1}{r^{2}} \frac{\partial}{\partial r}\left(r^{2} \kappa_{0} \frac{\partial T_{0}}{\partial r}\right)+\left[\frac{p_{0}}{\rho_{0}^{2}}-\left(\frac{\partial E}{\partial \rho}\right)_{T 0}\right] \frac{d \rho_{1}^{l}}{d t}
\end{gathered}
$$




$$
\begin{gathered}
+\left[\frac{p_{1}^{l}}{\rho_{0}^{2}}-\frac{2 p_{0}}{\rho_{0}^{3}} \rho_{1}^{l}-\left(\frac{\partial E}{\partial \rho}\right)_{T 1}^{l}\right] \frac{d \rho_{0}}{d t} \\
+\frac{1}{\rho_{0}}\left\{\frac{\partial \kappa_{0}}{\partial r} \frac{\partial T_{1}^{l}}{\partial r}+\kappa_{0} \frac{1}{r^{2}} \frac{\partial}{\partial r}\left(r^{2} \frac{\partial T_{1}^{l}}{\partial r}\right)+\frac{\partial \kappa_{1}^{l}}{\partial r} \frac{\partial T_{0}}{\partial r}+\kappa_{1}^{l} \frac{1}{r^{2}} \frac{\partial}{\partial r}\left(r^{2} \frac{\partial T_{0}}{\partial r}\right)\right. \\
-\kappa_{0} \frac{l(l+1)}{r^{2}} T_{1}^{l}-\kappa_{0} \frac{\partial T_{0}}{\partial r}\left[\frac{2-l(l+1)}{r^{2}}-\frac{2}{r} \frac{\partial}{\partial r}+\frac{\partial^{2}}{\partial r^{2}}\right] B^{l} \\
\left.-2 \frac{\partial B^{l}}{\partial r} \frac{1}{r^{2}} \frac{\partial}{\partial r}\left(r^{2} \kappa_{0} \frac{\partial T_{0}}{\partial r}\right)+\dot{q}_{1}^{l}-\frac{\rho_{1}^{l}}{\rho_{0}} \dot{q}_{0}\right\} \\
p_{1}^{l}=\left(\frac{\partial p}{\partial T}\right)_{\rho 0} T_{1}^{l}+\left(\frac{\partial p}{\partial \rho}\right)_{T 0} \rho_{1}^{l}, \\
\kappa_{1}^{l}=\left(\frac{\partial \kappa}{\partial T}\right)_{\rho 0} T_{1}^{l}+\left(\frac{\partial \kappa}{\partial \rho}\right)_{T 0} \rho_{1}^{l}, \\
\dot{q}_{1}^{l}=\left(\frac{\partial \dot{q}}{\partial T}\right)_{\rho 0} T_{1}^{l}+\left(\frac{\partial \dot{q}}{\partial \rho}\right)_{T 0} \rho_{1}^{l}, \\
\left(\frac{\partial E}{\partial T}\right)_{\rho_{1}}^{l}=\left[\frac{\partial}{\partial T}\left(\frac{\partial E}{\partial T}\right)_{\rho}\right]_{\rho 0} T_{1}^{l}+\left[\frac{\partial}{\partial \rho}\left(\frac{\partial E}{\partial T}\right)_{\rho}\right]_{T 0} \rho_{1}^{l}, \\
\left(\frac{\partial E}{\partial \rho}\right)_{T 1}^{l}=\left[\frac{\partial}{\partial T}\left(\frac{\partial E}{\partial \rho}\right)_{T}\right]_{\rho 0} T_{1}^{l}+\left[\frac{\partial}{\partial \rho}\left(\frac{\partial E}{\partial \rho}\right)_{T}\right]_{T 0} \rho_{1}^{l} .
\end{gathered}
$$

Here $\rho_{1 I}^{l}$ is the initial density perturbation in mode $l$, and the following symbols are defined:

$$
\begin{gathered}
A^{l} \equiv v_{1 r}^{l, m}, \\
B^{l} \equiv X_{r}^{l, m}, \\
C^{l} \equiv\left[r^{2} \nabla_{\Omega} \cdot\left(\mathbf{v}_{1}^{L}-v_{0} \Omega\right]^{l, m},\right. \\
D^{l} \equiv\left[\nabla_{\Omega} \cdot \mathbf{X}\right]^{l, m} .
\end{gathered}
$$

Thus $A^{l}$ is the modal amplitude of the radial component of the Lagrangian first-order velocity and $B^{l}$ is the modal amplitude of the radial component of the displacement. The symbol $\Omega$ denotes the angular part of the displacement, so that, to first order,

$$
\mathbf{X}=X_{r} \mathbf{e}_{r}+r_{0} \boldsymbol{\Omega} .
$$

Then the Lagrangian first-order velocity is

$$
\mathbf{v}_{1}^{L}=\frac{d \mathbf{X}}{d t}=\dot{X}_{r} \mathbf{e}_{r}+r_{0} \dot{\boldsymbol{\Omega}}+v_{0} \boldsymbol{\Omega}
$$


The symbol $\nabla_{\Omega}$ represents the angular divergence:

$$
\nabla_{\Omega} \cdot \equiv \frac{1}{r} \frac{\partial}{\partial \boldsymbol{\Omega}} \cdot \equiv \frac{1}{r \sin \theta}\left[\frac{\partial}{\partial \theta}\left(\sin \theta \mathbf{e}_{\theta^{*}}\right)+\frac{\partial}{\partial \phi}\left(\mathbf{e}_{\phi^{\cdot}}\right)\right] .
$$

So we see that $C^{l}$ is proportional to the angular divergence of the first-order angular velocity $r_{0} \dot{\boldsymbol{\Omega}}$, since

$$
\mathbf{v}_{1}^{L}-v_{0} \boldsymbol{\Omega}=\dot{X}_{r} \mathbf{e}_{r}+r_{0} \dot{\boldsymbol{\Omega}},
$$

and the angular divergence of $\dot{X}_{r} \mathbf{e}_{r}$ vanishes. Finally, $D^{l}$ is the modal amplitude of the angular divergence of the displacement.

Scannapieco and Cranfill (1978) derived their first-order equations in a quite general form. Frequently, however, the first-order equations are specialized to the case of an idealgas equation of state, and the zeroth-order flow is taken to be self-similar [Kidder(1976), Hattori et al. (1986)] These approximations lead to considerable simplifications in the first-order equations. If the first-order flow is furthermore assumed to be incompressible (Hattori et al. 1986), then analytic solutions to the first-order motion can be found in some cases.

Linear single-mode instability calculations in a nonlinear radiation hydrodynamic code An approach that is more widely used today than linearized perturbation calculations is that of linear instability calculations in nonlinear codes. This approach was demonstrated by Verdon et al. (1982) and its accuracy for perturbed shock waves investigated by Munro (1989). Its application for assessing the effect of material mixing in ICF implosions was outlined by Haan (1989). The aim in these studies was to determine the evolution of the first-order flow in situations of interest in ICF, but instead of solving the linearized perturbed hydrodynamic equations, these investigators represent the first-order quantities within the nonlinear radiation-hydrodynamics calculation as infinitesimal perturbations on the zeroth-order flow. The nonlinear code is not itself modified to handle the infinitesimal perturbations, save for ensuring that the code computes extremely smooth zeroth-order solutions; this means that the solutions must be free of spurious "ringing" and numerical noise caused by artificial discontinuities in the code's physics approximations. The advantage of this approach is that all the physics of the nonlinear code (for example, multigroup radiation transport) is included in an effectively linearized calculation of the infinitesimal angular perturbation, without the need to develop and solve elaborate systems of linearized equations such as Eqs. (E-13) through (E-23). (After all, as complicated as that system is, it incorporates only a crude, grey-diffusion treatment of radiation transport. To use linearized transport equations for many photon groups would add many more equations.) A disadvantage of the Haan-Munro approach is that the nonlinear code may suffer from noisy numerical algorithms and limited machine precision. Furthermore, convergence with mesh refinement of the instability growth may be rather slow.

In this approach, to calculate the linear growth of a single spherical-harmonic instability mode, a narrow angular sector of the capsule, at the capsule's equator, is represented by the mesh. The width of the two-dimensional sector is one-half-wavelength of the mode. ("Wavelength" is not a well-defined concept for spherical harmonics, but is useful near the 
equator, where the Legendre functions are well approximated by sinusoids.) An infinitesimal displacement in radius, with sinusoidal angular dependence, is applied to the mesh at the interface of interest. The amplitude of the displacement, $\eta_{l, \text { max0}}$, is typically about 1 $\AA$, or about $10^{-7}$ of the capsule dimensions. Thus, even if the instability grows by several orders of magnitude, its effect on the zeroth-order flow is negligible, as required if this is to be truly a calculation of linear growth. Neighboring mesh lines are likewise perturbed, with an amplitude $\eta_{10}(r)$ that decreases away from the interface in accordance with the radial part of the solutions of Laplace's equation in spherical geometry:

$$
\eta_{10}(r)=A r^{l}+\frac{B}{r^{l+1}}
$$

for mode $l$. If the displacement amplitude is chosen to peak at the outer radius $r_{\text {out }}$ of a shell and vanish at the inner radius $r_{i n}$, then

$$
\eta_{l 0}(r)=\eta_{l, \max 0} \frac{\left(r / r_{i n}\right)^{l}-\left(r_{\text {in }} / r\right)^{l+1}}{\left(r_{\text {out }} / r_{\text {in }}\right)^{l}-\left(r_{\text {in }} / r_{\text {out }}\right)^{l+1}} .
$$

It is observed in numerical experiments that this form for $\eta_{10}(r)$ leads to more rapid convergence of the computed instability growth factor as the mesh is refined, compared to a linear variation of $\eta_{10}$ with $r$, for example.

After the perturbation has been applied to the mesh, the calculation is performed in the usual way up to some time of interest $t_{1}$, such as ignition. The amplitude of the radial mesh displacement is determined everywhere in the mesh, and its maximum value $\eta_{l, \text { max }}\left(t_{1}\right)$ identified; usually the amplitude is largest near the boundary between the hotspot and the cold fuel. The ratio $\eta_{l, \max }\left(t_{1}\right) / \eta_{l, \max 0} \equiv G_{l}$ is called the growth factor of mode $l$.

Next, the true initial amplitude $R_{l m, 0}$ of mode $(l, m)$ is determined from a spectral analysis of the surface of a real ICF capsule. The linear amplitude of mode $(l, m)$ at time $t_{1}$ is then $R_{l m}=G R_{l m, 0}$, since linear growth rates are independent of $m$. If this amplitude exceeds the saturation amplitude $\nu r / l^{2}$, where $r$ is the radius at which the amplitude reaches its maximum value, then Haan's (1989) post-saturation amplitude, Eq. (D-3), is used instead of the linear amplitude.

In a similar manner, amplitudes are determined for all modes of interest at time $t_{1}$. (Although we are assuming that the growth factors $G_{l}$ have been obtained via a linear perturbation calculation with a nonlinear code, they might equally well come from a linearized code of the sort described in the preceding section.) Then the rms roughness $\sigma$ is given by a quadrature sum of the amplitudes of the modes:

$$
\sigma^{2}=\frac{1}{4 \pi} \sum_{l>1} \sum_{m} R_{l m}^{2}
$$

The bubble amplitude (the distance of penetration of low-density material into high-density material) is taken to be $\sqrt{2} \sigma$, while the spike amplitude (the distance of penetration of high-density material into low-density material) is taken to be $\sqrt{2}(1+A) \sigma$, where $A$ is the Atwood number. Finally, a 1D calculation of the capsule's implosion is carried out in 
which the interface is uniformly mixed over the appropriate distances into the high-density and low-density materials at time $t_{1}$. In this way we arrive at the goal of the Haan-Munro technique, namely, a prediction of the effect of initial interface roughness on the capsule's performance.

\section{Nonlinear multimode calculations}

In this approach, one carries out a direct simulation of the effect of realistic interface roughness on a capsule's performance. The interface of interest is perturbed with a spectrum of many modes, with finite amplitudes rather than infinitesimal amplitudes. Thus the initial interface in the calculation is a fairly direct representation of what a real interface looks like, with certain qualifications mentioned below. The calculation is run through the time of thermonuclear reaction in the capsule's fuel, and the yield determined as a function of the initial $\sigma$ and spectral content of the interface roughness.

In order to satisfy the boundary condition that the mesh displacement attain an extremum at the angular boundaries of the mesh for each mode, it is necessary to use a 90-degree sector of the capsule, extending from the pole to the equator. Sufficiently fine angular zoning is required to ensure that the highest modes are accurately resolved, since in general zoning that is too coarse causes an underestimate of growth rate. Thus in this approach a large number of zones is required, driving up the computational expense.

This approach is appealing because of its conceptual simplicity, and because it is potentially a realistic picture of instability development and the effect of instabilities on an ICF capsule. However, in addition to its expense, it suffers to date from various shortcomings which limit its accuracy. For example, most existing nonlinear radiation hydrodynamic codes are two-dimensional, so that only axisymmetric modes (that is, spherical harmonics with $m=0$ ) can be represented. (It is in this sense that the initial interface shape is not quite realistic; real surfaces are not axisymmetric on the microscopic scale of perturbations.) In the nonlinear stage of instability, the growth rate begins to depend on $m$, and is greater for larger $m$. Omitting this three-dimensional effect leads again to an underestimate of the net growth rate of $\sigma$.

Furthermore, many existing nonlinear radiation hydrodynamic codes use a Lagrangian mesh, which can become significantly distorted for highly unstable flows. This means that the mesh must be rezoned, that is, that physical variables must be mapped onto a more regular mesh, at various times in the calculation. Rezoning introduces inaccuracies due to numerical diffusion. If, however, rezoning is kept to a minimum, other inaccuracies occur because of the failure of code algorithms on irregular meshes. To avoid these difficulties, one might consider using an Eulerian code, in which the mesh is constrained to be regular and orthogonal at all times.

For all these reasons it is important to calibrate such calculations against data from experiments. It is also important to refine the technique so as to avoid the most obvious shortcomings, namely two-dimensionality and Lagrangian mesh distortion. Thus it seems that a fruitful direction for further research is the development of 3D Eulerian codes with high mesh resolution and appropriate physics, adequate for modeling real instability evolution with a minimum of simplifying approximations. 


\section{References}

Bodner S E, 1974, Rayleigh-Taylor instability and laser-pellet fusion, Phys Rev Lett 33761.

Chandrasekhar S, 1961, Hydrodynamic and Hydromagnetic Stability (Oxford, Glasgow).

Davies $\mathrm{R} M$ and Taylor G, 1950, The mechanics of large bubbles rising through extended liquids and through liquids in tubes, Proc R Soc London A 200375.

Diamond P, Eardley D, Katz J, Koonin S, Max C, Meiron D, Perkins F, and Rosenbluth M, 1993, Shock obliquity and stability in inertial confinement fusion, JASON report JSR-91-325, Mitre Corp, McLean, VA.

Gamaly E G, 1993, Hydrodynamic Instability of Target Implosion in ICF, in Nuclear Fusion by Inertial Confinement, eds Velarde G, Ronen Y, and Martínez-Val J M (CRC Press, Boca Raton).

Haan S W, 1989, Onset of nonlinear saturation for Rayleigh-Taylor growth in the presence of a full spectrum of modes, Phys Rev A 395812.

Hattori F, Takabe H, and Mima K, 1986, Rayleigh-Taylor instability in a spherically stagnating system, Phys Fluids 291719.

Lord Kelvin, 1910, Mathematical and Physical Papers, iv, Hydrodynamics and General Dynamics, Cambridge, England.

Kidder R E, 1976, Laser-driven compression of hollow shells: power requirements and stability limitations, Nucl Fusion 163.

Landau L D and Lifshitz E M, 1987, Fluid Mechanics (Pergamon, Oxford).

Layzer D, 1955, On the instability of superposed fluids in a gravitational field, Astrophys $J 1221$.

LeLevier R, Lasher G, and Bjorklund F, 1955, Effect of a density gradient on Taylor instability, Lawrence Radiation Laboratory (Livermore, CA) report UCRL-4459.

Lindl J D and Mead W C, 1975, Two-dimensional simulation of fluid instability in laser-fusion pellets, Phys Rev Lett 341273.

Lindl J D, 1988, Introduction to the physics of ICF capsules, in Inertial Confinement Fusion: Proceedings of the Course and Workshop held at Villa Monastero, Varenna, Italy, eds Caruso $\mathrm{A}$ and Sindoni $\mathrm{E}$ (Società Italiana di Fisica, Bologna).

McCrory R L, Morse R L, and Taggart K A, 1977, Growth and saturation of instability of spherical implosions driven by laser or charged particle beams, Nucl Sci and Eng 64 163.

Mikaelian K O, 1986, Approximate treatment of density gradients in Rayleigh-Taylor instabilities, Phys Rev A 331216.

Munro D H, 1989, Rippled shock front solutions for testing hydrodynamic stability simulations, Phys Fluids B1 134.

Ofer D, Shvarts D, Zinamon Z, and Orszag S A, 1992, Mode coupling in nonlinear Rayleigh-Taylor instability, Phys Fluids B 43549.

Richtmyer R D, 1960, Taylor instability in shock acceleration of compressible fluids, Commun Pure Appl Math 13297.

Scannapieco A J and Cranfill C W, 1978, A derivation of the physical equations solved in the inertial confinement stability code DOC, Los Alamos National Laboratory report 


\section{LA-7214-MS.}

Shvarts, D, 1994, Nonlinear evolution of multimode Rayleigh-Taylor and RichtmyerMeshkov instabilities in two and three dimensions, Bull APS 391576.

Takabe H, Mima K, Montierth L, and Morse R L, 1985, Self-consistent growth rate of the Rayleigh-Taylor instability in an ablatively accelerating plasma, Phys Fluids 283676.

Taylor G, 1950, The instability of liquid surfaces when accelerated in a direction perpendicular to their planes, Proc Roy Soc (London) A 201192.

Verdon C P, McCrory R L, Morse R L, Baker G R, Meiron D I, and Orszag S A, 1982, Nonlinear effects of multifrequency hydrodynamic instabilities on ablatively accelerated thin shells, Phys Fluids 251653. 